\title{
Design and numerical simulation of ejector nozzles for very small turbojet engines
}

\author{
R. Schmidt ${ }^{1}{ }^{\circ} \cdot$ A. Hupfer $^{1}$
}

Received: 18 February 2021 / Revised: 29 June 2021 / Accepted: 19 July 2021 / Published online: 16 August 2021

(c) The Author(s) 2021

\begin{abstract}
The examination of small turbojet engines for unmanned aviation systems is a main research activity at the Institute of Aeronautical Engineering at the Universität der Bundeswehr München. The special requirements for the design of these propulsion systems include compact installation size, high reliability and low acquisition costs. But the low overall efficiency, the high exhaust gas temperature and excessive noise emission limit the options for integration and mission profiles. The concept of the ejector nozzles offers a solution for further improving the propulsion system following the requirements. The secondary mass flow delivered from the ejector nozzle can not only reduce the exhaust gas temperature but also enhance the thrust. Ejector nozzles have been developed in combination with special mixer nozzles with the design goal of increasing the pumping performance and thrust augmentation. Both numerical and experimental investigations are conducted to study the mixing ability of the primary nozzle, the key to optimising the ejector performance.
\end{abstract}

Keywords Small gas turbines $\cdot$ Nozzle flow $\cdot$ Thrust augmentation $\cdot$ CFX

$\begin{array}{ll}\text { Abbreviations } \\ A_{\text {DIF }} & \text { Ejector diffusor area } \\ A_{\mathrm{I}} & \text { Ejector secondary inlet area } \\ A_{\mathrm{M}} & \text { Ejector area } \\ A_{\text {measure }} & \text { Area measurement plane secondary inlet } \\ A_{\mathrm{p}} & \text { Primary nozzle area } \\ A_{\mathrm{S}} & \text { Secondary flow area ejector nozzle } \\ c & \text { Velocity } \\ D & \text { Ejector shroud diameter } \\ F & \text { Thrust } \\ h & \text { Height } \\ \text { idex il } & \text { Inward lobe } \\ \text { index 0 } & \text { Ambient condition } \\ \text { index } 9 & \text { Nozzle exit plane } \\ \text { index EJ } & \text { Ejector inlet } \\ \text { index i } & \text { Inside position } \\ \text { index m } & \text { Middle position } \\ \text { index o } & \text { Outside position } \\ \text { index ol } & \text { Outward lobe } \\ L & \text { Ejector shroud length }\end{array}$

R. Schmidt

ralf.schmidt@unibw.de

1 Institute of Aeronautical Engineering, Universität d. Bundeswehr München, Werner-Heisenberg-Weg 39, 85577 Neubiberg, Germany

$\begin{array}{ll}L_{1} & \text { Length of the lobe } \\ \dot{m}_{\mathrm{p}} & \text { Mass flow turbojet engine } \\ r & \text { Radius } \\ r_{\mathrm{c} 5} & \text { Casing radius nozzle connection flange } \\ r_{\alpha} & \text { Lobe shape radius outward lobe } \\ r_{\beta} & \text { Lobe shape radius inward lobe } \\ T_{\mathrm{t} 5} & \text { Turbine outlet temperature } \\ \alpha & \text { Area ratio ejector nozzle } \\ \beta_{\mathrm{III}} & \text { Lobe shape angle inward lobe } \\ \beta_{\mathrm{mix}} & \text { Degree of mixing } \\ \Theta, \alpha \mathrm{III} & \text { Lobe mixer segment angle } \\ \Pi_{\mathrm{D}} & \text { Nozzle pressure ratio } \\ \sigma & \text { Ending angle lobe } \\ \Phi & \text { Thrust augmentation } \\ \varphi & \text { Lobe angle } \\ \varphi_{\mathrm{DIF}} & \text { Ejector diffusor half angle }\end{array}$

\section{Introduction}

Nowadays, very small turbojet engines with a thrust range of up to $1000 \mathrm{~N}$ are used in many applications. All these engines have a similar structural design. A single-stage centrifugal compressor, usually made of an aluminium alloy, delivers a pressure ratio of 2-4 [1]. The relatively large combustion chamber works with evaporator sticks [2]. The single-stage, solid cast axial turbine consists of a nickel-based alloy and 
provides the power for the compressor. The simple construction in combination with the small profile thickness of the turbine blades does not allow any internally cooled turbine components in these engines, which means that the turbine inlet temperature is limited to $1000{ }^{\circ} \mathrm{C}$ [3].

Engines of this configuration achieve a thrust-to-weight ratio of 6-10 and a thrust-specific consumption TSFC of $40-60 \mathrm{~g} / \mathrm{kNs}$ [3]. The current average acquisition costs of small turbojet engines are about $20 € / \mathrm{N}$. This is a mean value from the manufacturer's information for commercially available very small turbojet engines up to $1000 \mathrm{~N}$.

In addition to model aircraft propulsion, the areas of application for very small turbojet engines are primarily military reconnaissance and target drones such as the Airbus Do-DT25. Future applications like autonomous area reconnaissance or air freight delivery place even more demanding requirements on propulsion systems [4]. Consequently, very small turbojet engines must be improved in terms of their low overall efficiency (5-10\% [3]) and for integration into the aircraft. An improvement of the thermal efficiency calls for a higher pressure ratio and a higher turbine inlet temperature. Advanced materials like titanium, high-performance nickel alloy and ceramics [3] would be necessary with an enormous impact on the costs of acquisition. The other way would be to increase the mass flow $\dot{m}$, generating more thrust. The thrust $F$ resulting from the turbojet's nozzle expanding to ambient conditions $\left(c_{0}\right)$ is far away from the ideal thrust when the total potential energy is converted into kinetic energy. Equations (1) and (2) show that a higher mass flow generates a slightly lower $c_{\text {Nozzle }}$ but more thrust $F$ at given gas turbine power $P$ [5].

$F=\dot{m}\left(c_{\text {Nozzle }}-c_{0}\right)=\dot{m} \Delta c$

$P=\dot{m}\left(\frac{c_{\text {Nozzle }}^{2}}{2}-\frac{c_{0}^{2}}{2}\right)=F \cdot \frac{\left(c_{\text {Nozzle }}+c_{0}\right)}{2}$

To pump an additional mass flow, one can use pressure forces, as the fan stage of a turbofan engine does. However, the design approach of a turbofan engine cannot be used for very small gas turbines due to the limited opportunities for component downscaling. The manufacturing requirements would also make such a concept too complex and expensive.

The concept of the ejector nozzle [5-7] instead uses viscous forces to pump a secondary mass flow. Beside the thrust augmentation, the secondary mass flow of the ejector nozzle offers additional potential for exhaust gas cooling [8], IR-suppression [9] and noise emission reduction [10].

\section{Development of nozzles for very small turbojet engines}

The ejector nozzle (Fig. 1) works on the principle of a jet pump and has no moving parts. That makes it compact, lightweight, and reliable [11]. A fast-flowing propellant induces low-pressure areas and sucks in the pumped medium through a nozzle-shaped inlet. The pumped medium is accelerated in the mixing tube by momentum exchange. Ideally, the total flow leaves the mixing tube with a uniform speed profile. The pumping rate of a secondary mass flow is driven by the difference in speed between the propellant (exhaust gas jet) and the pumped medium (ambient air). The small turbojet engines with a jet speed of up to $550 \mathrm{~m} / \mathrm{s}$ are particularly suitable for the ejector nozzle concept. The thrust augmentation decreases significantly with increasing airspeed $c_{0}$, so that the concept mainly causes an increase in thrust during take-off and lowspeed flight [11]. The goal of the ejector nozzle concept is to maximise the thrust augmentation and to conserve the power density of the turbojet engine [6].

Therefore, a primary nozzle is developed with good mixing ability and low-pressure losses.

\subsection{Design and construction: ejector nozzle}

Ejector nozzles for thrust engines have already been designed for various applications. These include lifting engines for hovering flight [11] and thrust augmenting systems with noise reduction for business jets [7].

The design of the ejector nozzle is mainly determined by the parameters area ratio $\alpha$ and the mixing speed [12]. The area ratio $\alpha$ of the ejector nozzle is defined as:

$\alpha=\frac{A_{\mathrm{s}}}{A_{\mathrm{p}}}=\frac{A_{\mathrm{M}}-A_{\mathrm{p}}}{A_{\mathrm{p}}}=\frac{A_{\mathrm{M}}}{A_{\mathrm{p}}}-1$

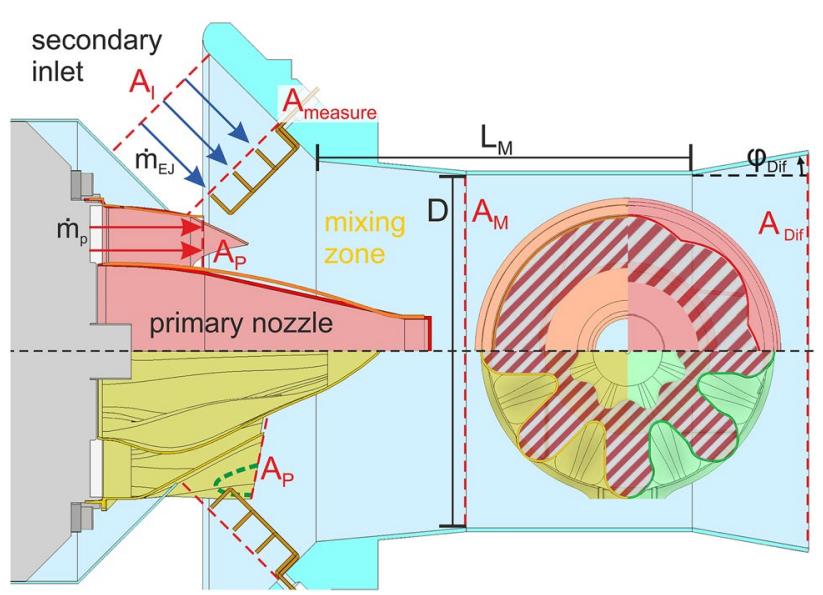

Fig. 1 Four tested primary and ejector nozzle configurations 
With the secondary flow area $A_{\mathrm{s}}$ and the primary nozzle area $A_{\mathrm{p}}$. The area $A_{\mathrm{s}}$ is the difference between the ejector shroud area $A_{\mathrm{M}}$ and $A_{\mathrm{p}}$. A theoretical thrust gain $\Phi(\alpha)$ is formulated with the equation for momentum conservation and the continuity equation [13]:

$\phi=\frac{\left(\alpha^{2}-1\right)(1-\alpha+\alpha \sqrt{2 \alpha})}{\left(\alpha^{2}+1\right)(\alpha-1)+2\left(\alpha^{2}+1\right)-2(1-\alpha+\alpha \sqrt{2 \alpha})(1+\alpha)}$

The equation for the ideal thrust augmentation results in a theoretical maximum of $\Phi$ for an infinite value $\alpha$. The weight and the ram drag limit the size for use on an aircraft engine [11]. These boundary conditions result in an optimal size for the combination of ejector nozzle, small turbojet engine, and application.

The mixing speed induced by the primary nozzle determines the L/D ratio of the ejector shroud (see Fig. 1). Complete mixing of the partial flows is essential for the ejector nozzle to work efficiently [12]. Theoretical considerations result in L/D ratios of 4-8 [11]. Forced mixers as primary nozzle can reduce the L/D ratio to values of 1 [12]. These forced mixers are related to the mixer for turbofan bypass mixing $[14,15]$. Experimental studies with so-called "scalloped lobe mixer nozzles" have confirmed short ejector nozzles with L/D ratios of 0.25 [12] due to the extremely high mixing speeds.

The inlet of the ejector nozzle has the crucial task of accelerating the ambient air into the mixing zone and generating the pumping low-pressure area [11]. The literature does not provide precise information on the ideal design of the inlet. High inlet area ratio $\left(A_{\mathrm{I}} / A_{\mathrm{s}}\right)$ of about $10-20$ are suggested in Ref. [11]. The form of the inlet should be optimised to reduce excessive losses when generating the low-pressure area.

The use of a diffuser at the ejector nozzle outlet increases the pump rate $[16,17]$. A positive influence on thrust augmentation depends on maintaining good mixing. Experimental investigations [11] suggested a maximal practical area ratio $A_{\mathrm{DIF}} / A_{\mathrm{M}}$ of 2 with a half-opening angles $\varphi_{\mathrm{DIF}}$ of $10^{\circ}$. Larger half-opening angle up to $30^{\circ}[16,17]$ can work with a high degree of turbulence because of the mixing. If the mixing is incomplete, the diffuser has no effect or losses due to flow separation [11]. For this reason, a diffuser is often dispensed on applications for thrust augmentation.

\subsection{Design and construction: lobe mixer nozzle}

The lobe mixer nozzles for use in an ejector nozzle differ from turbofan bypass mixers in some points: The meander-shaped outlet contour is also producing a very high degree of mixing. But the primary nozzle in the ejector nozzle must also take over the flow acceleration.

\subsubsection{Preliminary design: longitudinal path of the lobe}

For the geometrical design, the casing radius $r_{\mathrm{c} 5}$ of the nozzle connection flange and the inner radius of the ejector shroud $R_{\mathrm{M}}$ are assumed as input parameters (Fig. 2). The maximum outward lobe height $h_{\mathrm{ol}}$ should take up about half of the distance $d R_{\mathrm{EJ}}$ [17]. This ensures sufficient distance to fully utilize the ejector shroud. On the other hand, the ejector shroud is protected from direct contact with the hot exhaust gas.

The degree of penetration PEN or the total height of the lobe $h_{1}$ can be selected freely. The penetration PEN describes the mixing tube cross-section area that is penetrated by the height of the lobe $h_{1}$. In the study [16] the increase of penetration from 30 to $60 \%$ showed a steady increase in the pumping rate. The length $L_{1}$ of the lobe can either be specified directly or indirectly by specifying the lobe angle $\varphi_{\mathrm{ol}}$. This is recommended since the outward lobe is at risk of flow separation. Lobe angles $\varphi_{\mathrm{ol}}$ were tested from $9^{\circ}$ to $30^{\circ}$ for lobe design [12]. The total height of the lobe $h_{1}$ or the penetration PEN can then be varied until a constructively suitable value for $\varphi_{\mathrm{il}}$ is reached ( $15^{\circ}-45^{\circ}$ according to [12]). With a total height of $h_{1}=2 \cdot h_{\mathrm{o}}$, the lobe is symmetrical, otherwise it is asymmetrical.

\subsubsection{Advanced longitudinal path of the lobe}

A discontinuing contour at the transition into and out of the lobe should be avoided due to flow losses. The straight outward path is replaced by a combination of radius-line-radius (Fig. 2). This results in further construction parameters. One of the radii $\left(r_{1 \mathrm{ol}}, r_{1 \mathrm{il}}\right)$ can be freely selected. The second

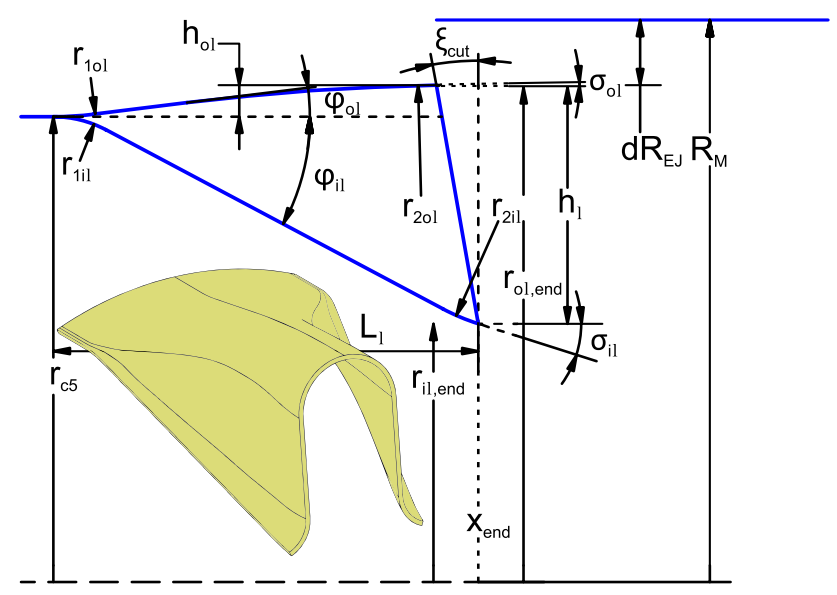

Fig. 2 Optimised longitudinal path of the lobe 
radius $\left(r_{2 \mathrm{ol}}, r_{2 \mathrm{i}}\right)$ enables an ending angle $\left(\sigma_{\mathrm{ol}}, \sigma_{\mathrm{il}}\right)$ that deviates from the actual lobe angle $\left(\varphi_{\mathrm{ol}}, \varphi_{\mathrm{il}}\right)$. The parameter $\sigma_{\mathrm{ol}}$ controls the distance to the point where the primary flow hits the ejector shroud. The value should only be changed in the single-digit range. The inward ending angle $\sigma_{\mathrm{il}}$ is based on the angle of the inner nozzle cone. There is also a cutback angle $\xi_{\text {cut }}$ for asymmetrical lobes. This reduces the flow surface of the lobe mixer nozzle and friction losses. The design of the lobe path contains further variation possibilities so that the final geometric shape is also based on empirical values. In general, the transition radii $\left(r_{1 \mathrm{o}}, r_{1 \mathrm{il}}\right)$ are specified first to prevent flow separation when entering the lobe passage.

The less critical end radii $\left(r_{2 \mathrm{ol}}, r_{2 \mathrm{il}}\right)$ are determined in this process. Finally, two path functions $r_{\mathrm{ol}}(x)$ and $r_{\mathrm{il}}(x)$ describe the outwards and inwards lobe.

\subsubsection{Design of the lobe mixer outlet shape}

Two radii and a connecting straight line form half of the lobe cross-section geometry (Fig. 3). The radii $r_{\mathrm{ol}}$ and $r_{\mathrm{il}}$ are given by the longitudinal paths $r_{\mathrm{ol}}(x)$ and $r_{\mathrm{il}}(x)$. The segment angle $\Theta$ is given by the number of lobes. The angle $\beta_{\mathrm{III}}$ determines the orientation of the flank of the lobe. Lobe contours are classified into three categories: The parallel lobe has its lobe flank parallel to the symmetry line $\left(\beta_{\mathrm{III}}=90^{\circ}-\Theta\right)$. With a higher value of $\beta_{\mathrm{III}}$,

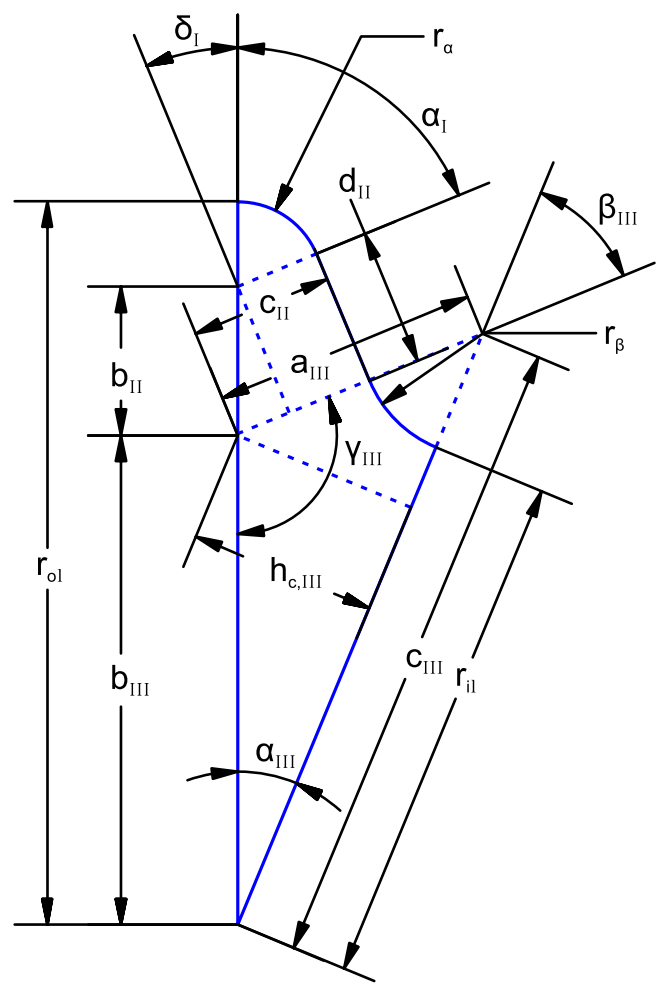

the outlet shape is referred as loop lobe LL. The opposite is the star lobe form SL. These outlet shapes have different effects on the radial distribution of the mixing (see Sect. 6.2.3).

\subsubsection{Transition from circular to lobe-shaped cross-section}

The cross-sectional geometry of a half lobe contour is split into several sub-elements with triangles and rectangles (Fig. 3). Because of the geometric constraints, the shape is determined by five values. The lobe-shaped cross-section's geometric relationships are determined by specifying $r_{\mathrm{o}}$, $r_{\mathrm{il}}, \Theta, \beta_{\mathrm{III}}$ and $r_{\alpha}$. The remaining lengths and angles can be calculated using the geometric conditions of a general triangle. For $r_{\mathrm{ol}}=r_{\mathrm{i} 1}$ and $r_{\alpha}=r_{\mathrm{c} 5}$, the geometric shape in Fig. 3 is reduced to the segment of a circle. For the transition from the circular to the lobe-shaped geometry several cross-sections are lined up. The transformation is given by the functions $r_{\mathrm{ol}}(x), r_{\mathrm{il}}(x), \beta_{\mathrm{III}}(x)$ and $r_{\mathrm{\alpha}}(x)$. The function for $\beta_{\text {III }}(x)$ is chosen to fulfil the conditions shown in Table 1. The end value $\beta_{\mathrm{III}}\left(x_{\text {end }}\right)$ gives the lobe mixer outlet shape (see Sect. 2.2.3). The more suitable but mathematically complex way to construct these nozzles is to set up a function for the cross-section value $A(x)$ and solve the equation system to get $r_{\alpha}(x)$.

\subsubsection{Contoured inner cone}

Instead of a rotationally symmetrical inner cone, a contoured inner cone can be used. This creates a better split into multiple mixing channels. A meander-shaped pattern is also used for the contour. The outward and inward lobe of the nozzle shroud and inner cone can be arranged in phase or $\Theta$ off phase. A study [8] for rectangular forced mixer lobes found that the vortex system created by staggered lobes is slightly better for mixing. The longitudinal path and cross-sectional contours are designed in the same way as the outer liner of the lobe mixer nozzle.

Table 1 Conditions for valid geometry

\begin{tabular}{|c|c|}
\hline Parameter & Equation \\
\hline Positive $d_{\mathrm{II}}$ & $d_{\mathrm{II}} \geq 0$ \\
\hline Limits $r_{\alpha}$ & $r_{\alpha, \min }<r_{\alpha}<r_{\alpha, \max }$ \\
\hline $\begin{array}{l}\text { Minimal } r_{\alpha} \\
\Rightarrow \beta_{\mathrm{III}}=0\end{array}$ & $r_{\alpha, \min }=\frac{r_{\mathrm{il}}-r_{\mathrm{ol}} \cos \alpha_{\mathrm{III}}}{1-\cos \alpha_{\mathrm{III}}}$ \\
\hline $\begin{array}{l}\text { Maximal } r_{\alpha} \\
\Rightarrow r_{\beta}=0\end{array}$ & 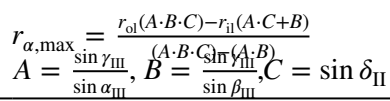 \\
\hline
\end{tabular}

Fig. 3 Calculation of the geometry parameters 


\section{Experimental investigation setup}

Extensive experimental tests were carried out in preparation of the numerical investigations. The experiments are intended to provide a database for determining the boundary conditions. The simulation is adapted and verified with the measured values. Consequently, an ejector nozzle concept was developed [6] and tested with a $180 \mathrm{~N}$ turbojet engine.

\subsection{Investigated primary nozzles on a $180 \mathrm{~N}$ turbojet engine}

The series of tests examined the effects of different primary nozzle types (Fig. 4). Each type was tested with and without the ejector nozzle. The classic thrust nozzle (1) has an annular outlet cross-section and is referred to here as the standard nozzle SN. Nozzle (2) is known as the chevron nozzle CVN. This type of nozzle is mainly used in aviation because of its ability to reduce noise emission. The lobe mixer nozzle LMN offers great potential $[16,17]$ in combination with an ejector nozzle. Two prototypes were developed for the $180 \mathrm{~N}$ turbojet engine. The design of both is based on parameters from the literature $[12,16]$ and empirical geometry values of a previous lobe mixer nozzle concept [6]. The lobe mixer nozzle (4) LMN-SC has additional cut-outs on the lobe flanks to enhance the mixing speed according to Ref. [12]. The lobe mixer nozzles were numerically adapted to the operating parameter of the standard nozzle SN.

\subsection{Combination with the ejector nozzle}

An ejector nozzle was designed to study the correlation between the primary nozzle, mixing speed, and thrust augmentation. The ejector tests were done with different configurations (Fig. 1). The ejector nozzle is made for compact size with an area ratio $\alpha$ of 1.77 . The ejector inlet is designed with an area ratio $A_{\mathrm{I}} / A_{\mathrm{s}}$ of 2.5 to keep the inlet velocity low. The short mixing length is acceptable for the expected high mixing speed of the lobe mixer nozzles [12]. An outlet diffusor could be attached at the end of the ejector nozzle, which has a moderate area ratio of 1.25 and a half-opening angle

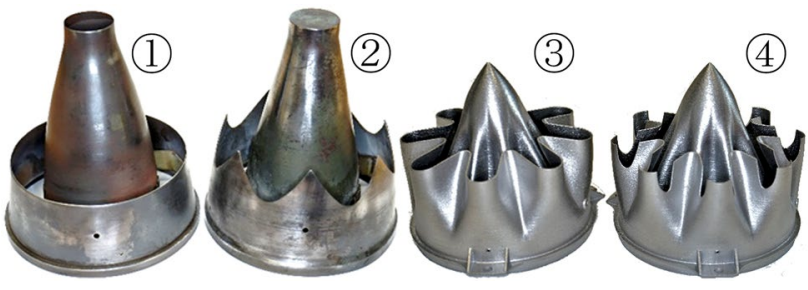

Fig. 4 (1)SN, (2) CVN, (3LMN, (4LMN-SC of $10^{\circ}$ [11]. This makes it possible to test the effect of the diffusor on the thrust augmentation.

\subsection{Measurement equipment}

The institute owns a special test bench for very small turbojet engines up to $500 \mathrm{~N}$ of thrust. Existing engines are tested with comprehensively installed measurement technology and new developments can be analysed.

\subsubsection{Test bench for very small turbojet engines}

On the test bench, a variety of different sensor types measure thrust $F$, fuel consumption $\dot{m}_{\text {fuel }}$, rotational speed, and compressor pressure ratio. The turbine outlet temperature $T_{\mathrm{t} 5}$ is measured with four thermocouples distributed around the circumference of the turbine outlet (index 5). A standard inlet nozzle for volume flow measurement according to ISO 5801 is attached to the engine. The laboratory with the test bench features a temperature-controlled ventilation system and an exhaust chimney to keep the test conditions constant and reproducible.

\subsection{2 $360^{\circ}$ profile measurement system for jet flow}

A $360^{\circ}$ profile measuring system was developed to provide high-resolution temperature measurements in the exhaust gas jet. These values are needed to gather information on the mixing progress. The system contains four probes attached to a rotatable ring at $90^{\circ}$ intervals. Three thermocouples $\left(T_{\mathrm{t} 9 \mathrm{i}}, T_{\mathrm{t} 9 \mathrm{~m}}, T_{\mathrm{t} 9 \mathrm{o}}\right)$ are mounted at different radial depths on each probe. A position-controlled servo motor rotates the ring while the engine is running.

The probes are rotated further in $5^{\circ}$ steps and the measurement data is recorded for this position after a short holding time, while the engine holds the load level. In total, the four probes are rotated by $90^{\circ}$ from their starting position. The $360^{\circ}$ profile of the temperatures in three radial depths of the nozzle flow is compound in the evaluation after the test. In addition, three total pressure tubes $\left(p_{\mathrm{t} 9 \mathrm{i}}, p_{\mathrm{t} 9 \mathrm{~m}}, p_{\mathrm{t} 9 \mathrm{o}}\right)$ with variable radial depth and a static edge pressure measurement $p_{9}$ were installed at each probe. These also follow the rotation of the measuring ring and makes it possible to create a profile of the dynamic pressure in three different radial depths of the nozzle flow $\left(\Delta p_{\mathrm{t} 9 \mathrm{i}}, \Delta p_{\mathrm{t} 9 \mathrm{~m}}, \Delta p_{\mathrm{t} 9 \mathrm{o}}\right)$. A master plate was $3 \mathrm{D}$-printed for the position adjustment of the probes. This ensures a high level of reproducibility of the measurement positions. The middle position (index $\mathrm{m}$ ) is placed on the area bisecting radius of the outlet crosssection. The inner radius (index i) halves the inner half of the cross-section, while the outer radius (index o) divides the outer half of the cross-section (see Fig. 27). 


\subsubsection{Measurement in the ejector inlet}

Pressure and temperature sensors are installed in the ejector inlet to collect information about the ejector secondary mass flow. Four static pressure holes $p_{\mathrm{EJ}}$ and four thermocouples $\mathrm{T}_{\mathrm{EJ}}$ are distributed in the inlet's measuring plane around the circumference (see Fig. 1). In addition, three total pressure tubes $\left(\Delta p_{\text {tEJ } \mathrm{i}}, \Delta p_{\mathrm{tEJ} \mathrm{m}}, \Delta p_{\mathrm{tEJ} \mathrm{o}_{\mathrm{o}}}\right)$ are placed at two circumferential positions $\left(0^{\circ}\right.$ and $\left.180^{\circ}\right)$. They provide information about the velocity profile in the inlet passage. The crosssection $A_{\text {measure }}$ is adjusted with a master plate during assembly. With the value of $A_{\text {measure, }}$, dynamic pressure profile, and density, the secondary mass flow was estimated.

\subsubsection{Optical setup}

The nozzle flow from the primary nozzle is also observed via a Schlieren optical setup. The Schlieren optics consists of a $Z$ arrangement with two $200 \mathrm{~mm}$ parabolic mirrors at a deflection angle of $3^{\circ}$. A $10-W$ LED with a light condenser lenses system is used as light source. The nozzle flow is filmed by a high-speed camera Photron AX200 Mini with $6400 \mathrm{fps}$ with a resolution of $1024 \times 1024$ pixels. Due to the high brightness in the measuring section, the shutter speed could be reduced to $1 / 200000 \mathrm{~s}$, so that the recording is not sensitive to stray light. Schlieren images were taken at five different load levels from idle to maximal thrust.

\subsubsection{Test procedure}

Each primary nozzle configuration was measured twice. In the first measurement, the $360^{\circ}$ profile of pressure and temperature was recorded near the nozzle outlet (near field). The Schlieren images were taken at the same time. In the second run, the profiles were measured at a distance to the nozzle outlet (far field). The position corresponds to the measuring position at the outlet of the ejector nozzles, which was installed later. The ejector nozzle, in combination with the different primary nozzles, was measured with and without the outlet diffuser. The profile measurement took place directly at the outlet of the ejector nozzle.

\section{Numerical simulation of nozzles for very small turbojet engines}

First, this numerical simulation is intended to provide information on optimising lobe mixer primary nozzle. Because of the difficult boundary conditions, the numerical setup is adjusted with the experimentally measured data. The simulation was performed with the CFX module from ANSYS.

\subsection{Physical mock-up}

The predefined material "air ideal" is used as flow medium in the simulation. Very small gas turbines have a fuel-to-air ratio about 1:40. As a result, the influence of fuel on the thermodynamic properties of the fluid is not considered. The material properties are extended with polynomial functions $f(T)$ for the specific heat capacity, the thermal conductivity and the dynamic viscosity, which approximate the experimental data of air according to Ref. [18] well. The turbulence is modelled with the implemented "Shear Stress Transport" (SST) model, which combines both the flow phenomena in the free jet and in the area close to the wall with an acceptable computational effort.

The "total energy model" is used for heat transport, which is specified as the standard model for the current flow with Ma $>0.3$ [19].

\subsection{Simulation geometry and boundary conditions}

Two different models were investigated and compared to experimental data, to find the best way of simulating the nozzle of a very small turbojet engine. The numerical preliminary examination was performed on the model of the standard nozzle SN. The nozzle flow was modelled as a free jet. The rotational symmetry was used and only a $45^{\circ}$ segment of the nozzle flow was calculated to reduce computing time. The simplified simulation domain (Fig. 5) only contains the nozzle. Exhaust gas from the turbine enters the nozzle and exits it into a volume. The data for the volumetric dimensions were taken from the literature [20]. It has a diameter of $3 \cdot r_{9}$ and a length of $14 \cdot r_{9}$ (see Fig. 5). The boundaries of the volume were treated as "opening".

The second extended simulation domain examined also includes the very small turbojet engine as a black box. At the compressor inlet the air mass flow exits the simulation domain and re-enters it into the nozzle with the values of exhaust gas. The surrounding volume has a diameter of five times the compressor inlet diameter.

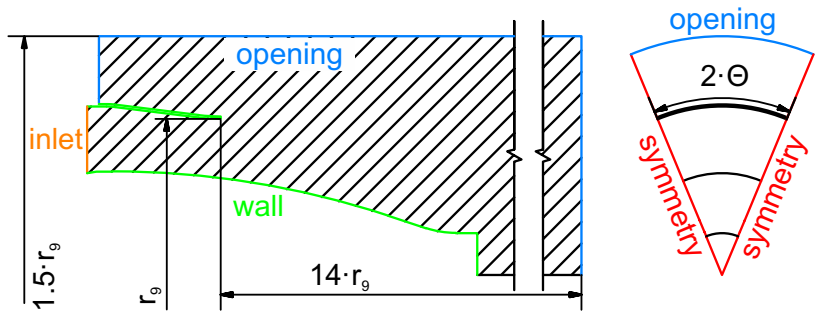

Fig. 5 Boundary conditions simplified simulation domain 
Table 2 Comparison of results from simulation and experimental measurements

\begin{tabular}{llr}
\hline Type of investigation & Thrust $F[N]$ & $\Pi_{\mathrm{D}}[-]$ \\
\hline Test SN & 195.5 & 1.65 \\
Simulation SN & $+0.7 \%$ & $-1.6 \%$ \\
Test CVN & 192.7 & 1.62 \\
Simulation CVN & $+0.5 \%$ & $-2.1 \%$ \\
Test LMN & 195.8 & 1.64 \\
Simulation LMN & $+0.3 \%$ & $-1.0 \%$ \\
Test LMN-SC & 190.1 & 1.61 \\
Simulation LMN-SC & $+0.6 \%$ & $-0.3 \%$ \\
\hline
\end{tabular}

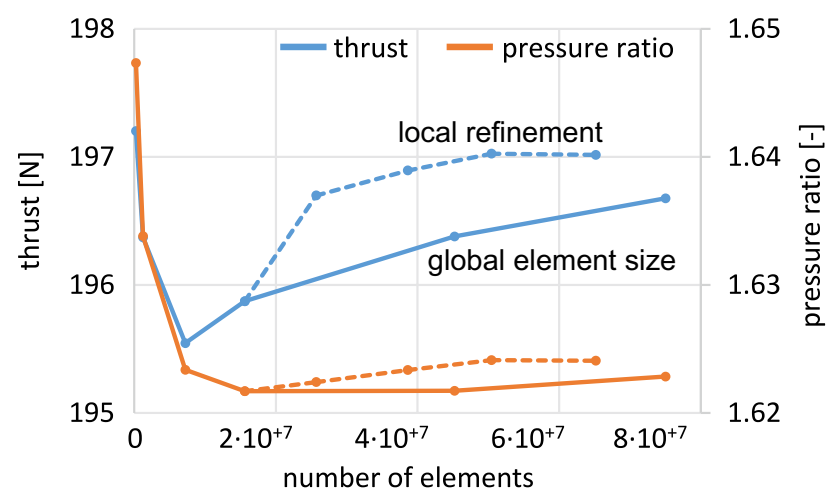

Fig. 6 Investigation of independent result of parameters thrust and nozzle pressure ratio

The input data for the mass flow $\dot{m}_{\mathrm{p}}$, turbine outlet temperature $T_{\mathrm{t} 5}$ and ambient conditions $\left(p_{0}\right.$ and $\left.T_{0}\right)$ was obtained from the experimental measurements of the $\mathrm{SN}$.

The simulation calculates the values for the thrust $F$ and the nozzle pressure ratio $\Pi_{\mathrm{D}}$, which are compared with the measured values (Table 2). Both simulation domains showed very similar results for thrust $F$ and nozzle pressure ratio $\Pi_{D}$. The simplified simulation domain achieved a good convergence with residuals smaller than $10^{-5}$ within 100 iterations. The extended simulation domain only achieved a similarly good result after 600 iterations. Therefore, the simplified model was used for all further simulations, as it enables results with good accuracy much faster.

\subsubsection{Meshing of the simulation domain}

The CFX module "Mesh" was used for meshing. Wall surfaces were covered with a prism layer with a first layer height of $4 \cdot 10^{-6} \mathrm{~m}$. The layer height was estimated using known flow data to get a $y+$ criterion less than 1 . The evaluation of the results shows that $y+$ could be kept below 1, covering a large area and did not exceed 3.1 locally.
The independence of the results from the mesh resolution is investigated using a series of simulations with a variation in element size and number (Fig. 6). The global element size was varied between $2 \cdot 10^{-3} \mathrm{~m}$ and $2.8 \cdot 10^{-4} \mathrm{~m}$.

The evaluated results of thrust $F$ and nozzle pressure ratio $\Pi_{D}$ hardly change below an element size of $5 \cdot 10^{-4} \mathrm{~m}$. However, the analysis of the flow profiles in the near nozzle outlet area indicates that an acceptable accuracy is only achieved with an element size below $2.1 \cdot 10^{-4} \mathrm{~m}$. Additional simulations were performed with a refinement of the elements up to $1.6 \cdot 10^{-4} \mathrm{~m}$ within the nozzle and near the outlet area.

The analysis identified a combination of a global element size of $10^{-3} \mathrm{~m}$ and a local refinement to $1.6 \cdot 10^{-4} \mathrm{~m}$ at near nozzle outlet as being particularly suitable. The numerical calculations could be performed within a reasonable calculation time and with a good quality of results.

\section{Verification of numerical simulation results by experimental measurement}

First, the experimental gathered performance data for thrust $F$ and nozzle pressure ratio $\Pi_{D}$ from the standard nozzle $\mathrm{SN}$, the chevron nozzle $\mathrm{CVN}$ and the two lobe mixer nozzles (LMN and LMN-SC) are compared with the simulation results in Table 2. The residual deviation between the numerical results and the measurement data are below the measurement tolerance of the experimental test setup. The total relative uncertainty for both thrust measurement and pressure ratio $\Pi_{D}$ is about $1.5 \%$. The performance data show the good agreement of the simulation model to the experiment.

Next, the temperature and total pressure profiles from the measurement are compared with the results of the numerical simulation. Since the temperature and total pressure profiles tend to be similar, the comparison is only carried out on the temperature data in this paper. The following temperature profiles from measurement and simulation are located on the "near field" (close to the nozzle outlet) and "far field" planes (outlet plane of the later ejector nozzle). These differ from the evaluation planes nozzle outlet P9 (used in Chapter 6) and one nozzle diameter distant 1DD due the limited accessibility in the experimental set up. The measurement data are combined into 2D plots and linearly interpolated for better visual analysis.

The $360^{\circ}$ measurement has a resolution of three radial values and an angular step width of $5^{\circ}$, which is significantly coarser than that of the simulation The plot resolution from the simulation was $0.1 \mathrm{~mm}$ in radial height and $0.5^{\circ}$ step size in the circumferential direction. The limited resolution and the interpolation of the measured data meant that strong gradients cannot be represented accurately. The used sheathed thermocouples of type $\mathrm{K}$ are positioned in the nozzle flow. 
Therefore, the temperature measured is slightly higher than the static temperature and below the total temperature. The difficult accessibility hinders the determination of the exact relationship or measurement with total temperature probes. The total temperature is calculated in the evaluation according to Refs. [21, 22] with a Mach number-dependent recovery factor between 0.8 and 0.85 . This causes a maximal deviation of $12{ }^{\circ} \mathrm{C}$.

The measurement uncertainty of the used thermocouples is $0.75 \%$ of the temperature value with a minimal deviation of $2.5^{\circ} \mathrm{C}$. In combination with the acquisition module, the maximal uncertainty is approx. $8{ }^{\circ} \mathrm{C}$.

The measurement uncertainty of the thermocouple is low compared to the measurement uncertainty due to the positioning accuracy. With temperature gradients of up to $400{ }^{\circ} \mathrm{C} / \mathrm{mm}$, the exact position of the measurement has a major impact on the measured temperature. The uncertainty due to the circumferential deviation of $2^{\circ}$ is shown in the form of error bars on the measured values in the diagrams. The radial and axial deviation is estimated to be $1 \mathrm{~mm}$. The maximum temperature deviation resulting from the combination of axial and radial positioning error is determined by the surrounding temperature gradients. The uncertainty therefore depends strongly on the measurement position. This aspect should be examined further in the future.

The verification of the numerical simulation with the temperature profiles is therefore mainly assessed based on the correct reproduction of the trend curves and distributions.

The degree of mixing $\beta_{\text {mix }}$ is calculated according to Ref. [16]. The integral is replaced by a summation because of the depreciation of the simulation domain:

$\beta_{\text {mix }}=\frac{\int c^{2} d A}{\bar{c}^{2}}=\frac{\sum_{i} c_{i}^{2} A_{i}}{\bar{c}^{2} A_{\text {ges }}}$

The degree of mixing $\beta_{\text {mix }}$ reaches the value 1 for complete mixing. In reality, this ideal state can only be approximated with a mixer nozzle.

\subsection{Analysis of the temperature profiles for primary nozzles}

\subsubsection{Standard nozzle SN}

The following Fig. 7 shows the near field 2D temperature profile of the standard nozzle $\mathrm{SN}$, which is created from the measurement data. The dashed lines mark the paths of the measuring probes. For comparison, a $45^{\circ}$ segment of the static temperature distribution from the numerical simulation is integrated.

Compared to the measurement, the simulation shows a significantly sharper transition from the nozzle flow to the ambient. The transition is also on a higher radius than the

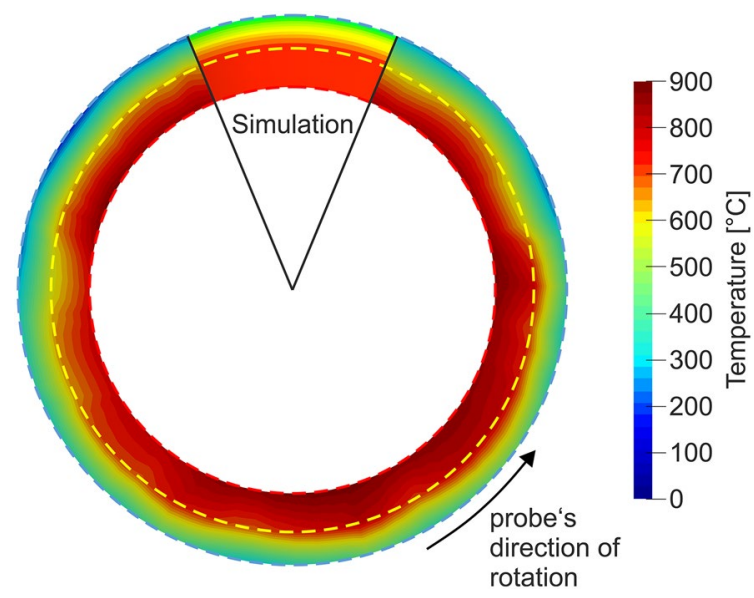

Fig. 7 Temperature distribution SN (near field): measured vs simulation

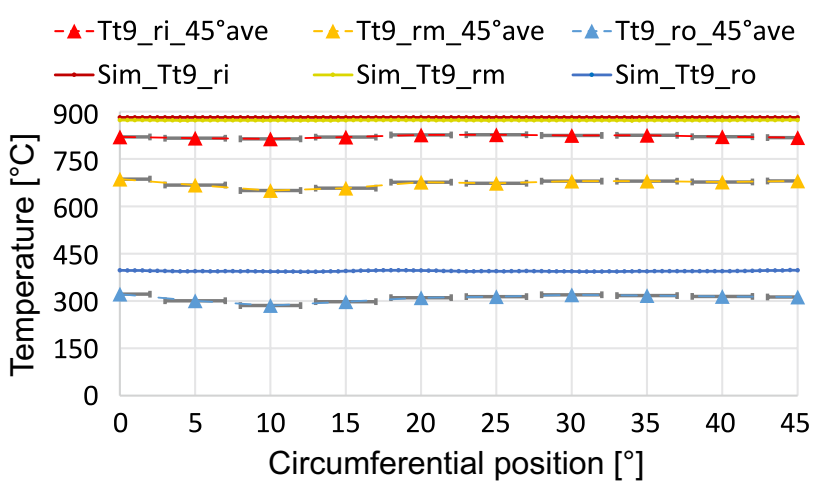

Fig. 8 Circumferential profile SN (near field): measured temperature vs simulation static temperature

measurement data suggests. The deviant representation of the measurement results from the limited resolution. The interpolation causes the softer transition. For a quantitative comparison of the temperatures' circumferential profiles, the measured $360^{\circ}$ profile is divided into the same number of segments that are used in the numerical simulation. The measurement data of all segments are averaged to compensate for local disturbances. The measured temperatures on radii $r_{\mathrm{m}}$ and $r_{\mathrm{i}}$ deviate slightly from the simulation data. Only in the case of the temperature on the outer radius $r_{\mathrm{o}}$ a larger discrepancy remains, as Fig. 8 shows.

Simulation and measurement contain mostly the same expected temperature curves for the standard nozzle. Due to the rotational symmetry of the standard nozzle, the temperature profiles have no gradients in the circumferential direction and a steady profile in the radial direction. The small surface of the nozzle flow keeps the potential for momentum exchange low. The effect can be assessed in the far field (Fig. 9). The mixing has reduced the radial gradients, but a noticeable difference remains. 


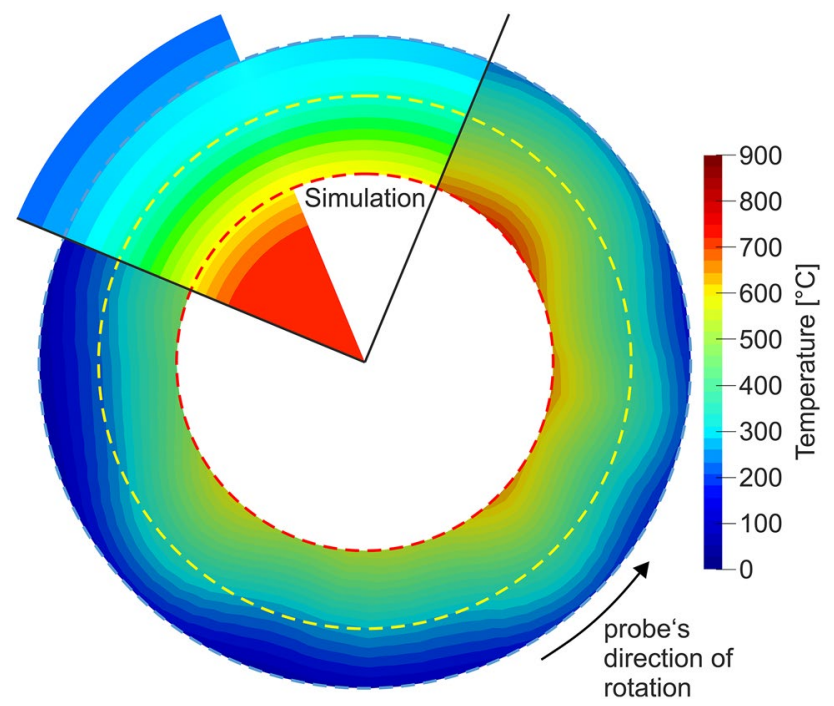

Fig. 9 Temperature distribution SN (far field): measured vs simulation

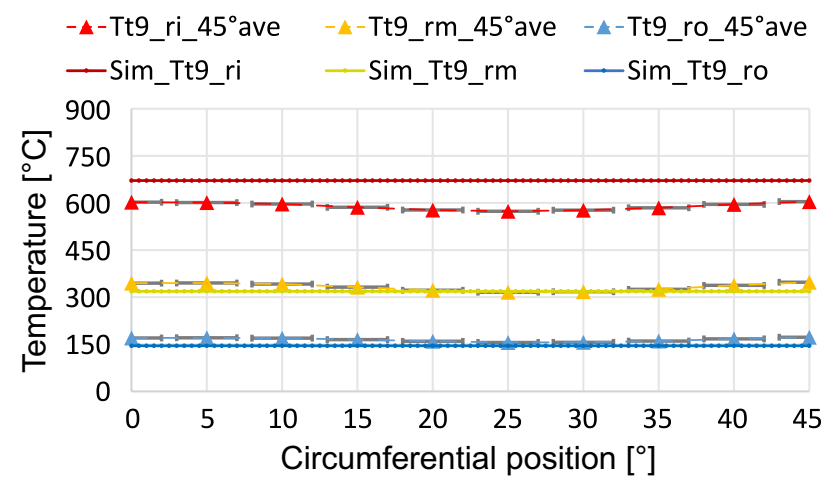

Fig. 10 Circumferential profile SN (far field): measured temperature vs simulation static temperature

The simulation also makes the hot core flow visible, what the measurement cannot. The high temperature in the core flow is an indication of a poor exchange with the outside area.

The circumference profiles (Fig. 10) from simulation hardly differ from the measurement. Both reproduce the tendency of the temperature curves equally.

Simulation and temperature measurement in the experiment arrive at the same result: the standard nozzle SN produces a uniform distribution in the circumferential direction. But the momentum cannot be shifted from the core flow to the outside area. The mixing capability is accordingly rated low. The degree of mixing $\beta_{\text {mix }}$ is calculated to 1.927 using the simulation data.

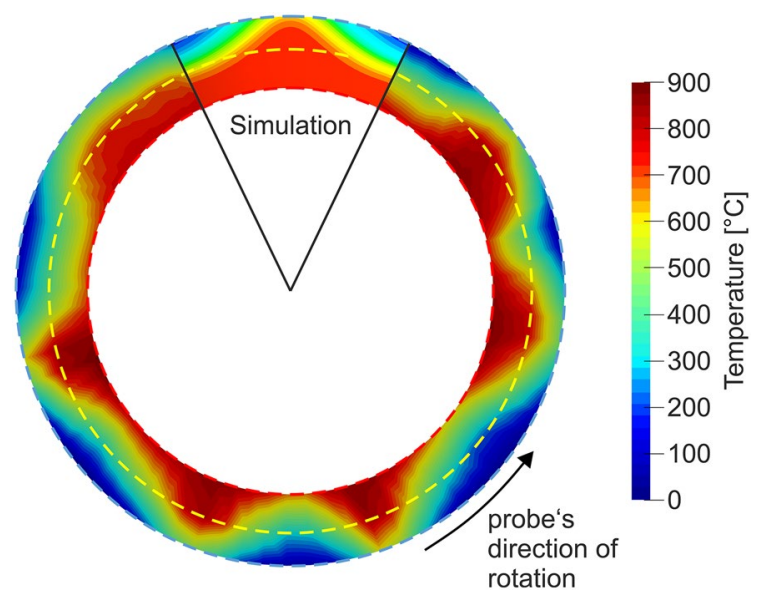

Fig. 11 Temperature distribution CVN (near field): measured vs simulation

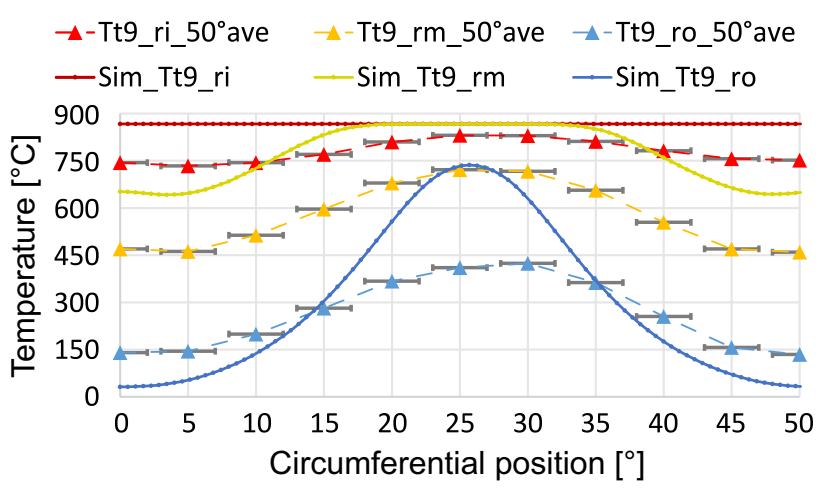

Fig. 12 Circumferential profile CVN (near field): measured temperature vs simulation static temperature

\subsubsection{Chevron nozzle CVN}

Figure 11 shows the measured temperature distribution at the outlet of the chevron nozzle. A $50^{\circ}$ segment with the plot of the static temperature from the simulation is inserted for comparison. In contrast to the standard nozzle SN, the seven spikes of the chevron nozzle CVN also generate gradients in the circumferential direction. The circumferential profiles of the temperature from measurement and simulation show similar behaviour, as shown in Fig. 12.

However, the measured data indicates that the exchange of momentum affects the core flow more strongly. One possible explanation is that the measuring plane was further away from the nozzle in the experiment than it has originally been intended. All temperature curves from the simulation tend to have a gradient in the circumferential direction. These are so small in the case of the profiles on the middle $r_{\mathrm{m}}$ and inner radius $r_{\mathrm{i}}$ that they are not visible in the representation. 


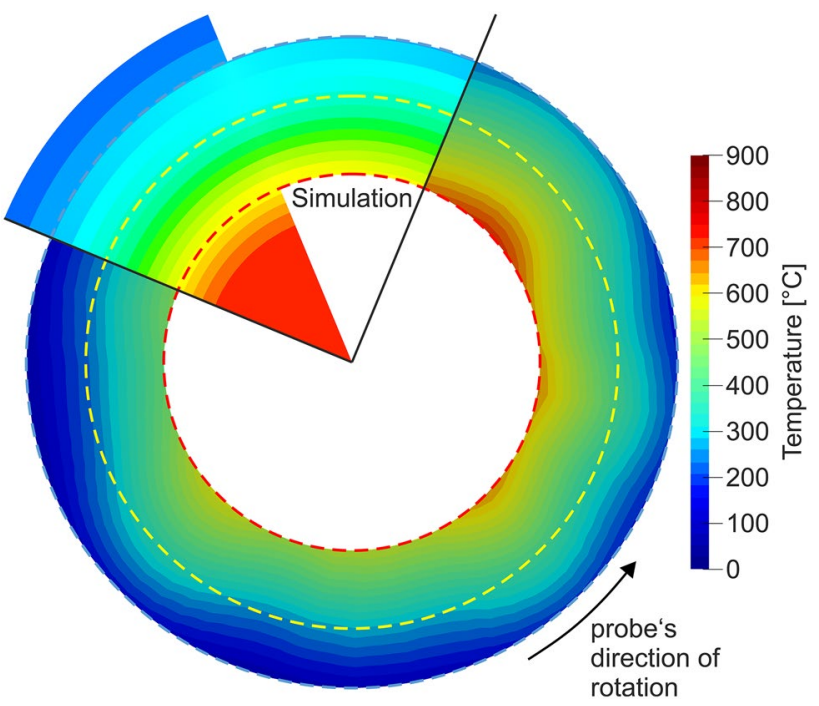

Fig. 13 Temperature distribution CVN (far field): measured vs simulation

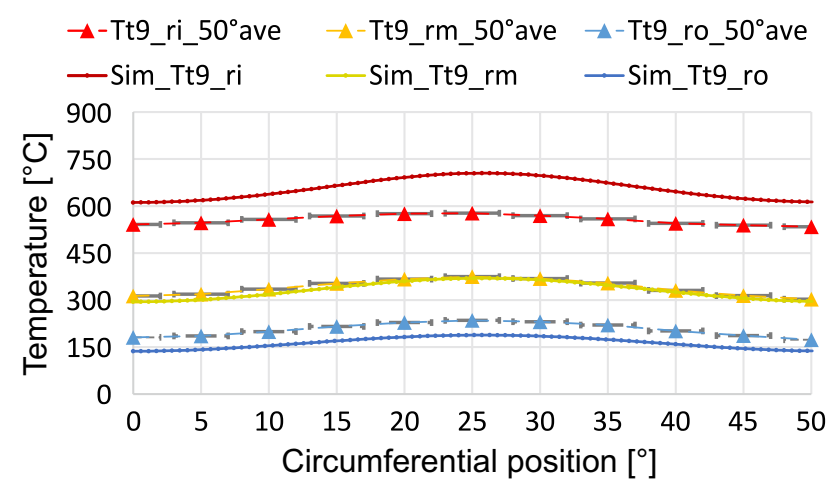

Fig. 14 Circumferential profile CVN (far field): measured temperature vs simulation static temperature

These gradients are more clearly visible in the map of the far field (Fig. 13).

The core flow is hardly recorded by the probe system at the chevron nozzle CVN too. The simulation data shows an increasing influence of the mixing on the core flow. The circumferential profiles from Fig. 14 still contain the gradients in the circumferential direction.

The profiles from simulation and measurement on the middle and outer radius are both similar in terms of the trend and the absolute value range. The temperature profile from the simulation on the inner radius $r_{\mathrm{i}}$ has a stronger gradient and higher temperature values. Correspondingly, the mixture is rated lower in the simulation.

Basically, the analysis of the chevron nozzle CVN shows higher deviations between simulation and measurement. One reason could be unsteady vortex systems caused by the

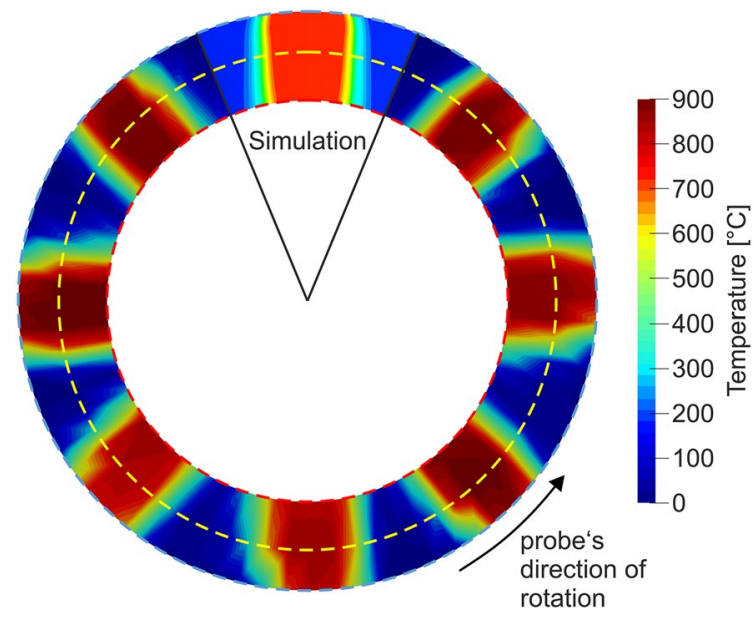

Fig. 15 Temperature distribution LMN (near field): measured vs simulation

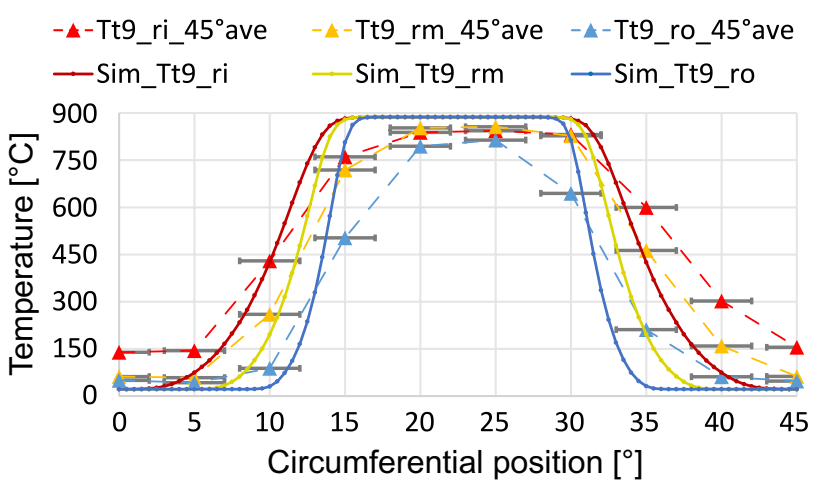

Fig. 16 Circumferential profile LMN (near field): measured temperature vs simulation static temperature

spikes. The Schlieren recordings suggest these vortex systems (see Sect. 5.2). These generate an increased exchange between the shear layer flow and the core flow. This phenomenon is not considered since a steady state is presumed in the simulation. Using the simulation data, the degree of mixing $\beta_{\text {mix }}$ is calculated at 1.737 .

\subsubsection{Lobe mixer nozzle LMN}

Figure 15 shows the measured temperature distribution at the outlet of the lobe mixer nozzle LMN. In addition, a $45^{\circ}$ segment of the static temperature distribution from the numerical simulation is added.

The comparison between simulation and measured temperature distribution again demonstrates the problem that strong gradients are weakened by the limited resolution in combination with the interpolation. Nevertheless, the simulation and measurement show the same formation of 
eight hot and cold zones, which are delimited by strong gradients. The graphic of the circumferential profiles in Fig. 16 shows that most of the measuring points are on the simulation curves. But there are too few measuring points around the strong gradients to describe this correctly.

The LMN lobe mixer nozzle generates extreme gradients where the temperature between two points with a distance of $2 \mathrm{~mm}$ increases by $800{ }^{\circ} \mathrm{C}$. The slow response of temperature measurement with thermocouples makes it difficult to track this correctly.

The temperature distribution in the far field (Fig. 17) shows good agreement between the temperature distribution from the simulation and the measured temperature profile.

The simulation provides better information on the core flow. This is strongly influenced by the mixing process. In contrast to the standard nozzle and the chevron nozzle, the hot area in the centre is smaller. The impulse is directed outwards as desired. The temperature curves in the circumferential direction (Fig. 18) confirm the good agreement between measurement and simulation. The range of values and the trend are both plotted similarly. The deviation of the maximum temperature on the inner radius $r_{\mathrm{i}}$ may be caused by the averaging of the measurement data on a $45^{\circ}$ segment.

Our analysis of the measurement and simulation data from the lobe mixer nozzle LMN comes to the same conclusion: the lobes have a stronger influence on the core flow. The mixing in the middle and outside area is in an advanced state. The degree of mixing $\beta_{\text {mix }}$ is calculated to 1.319 using the simulation data.

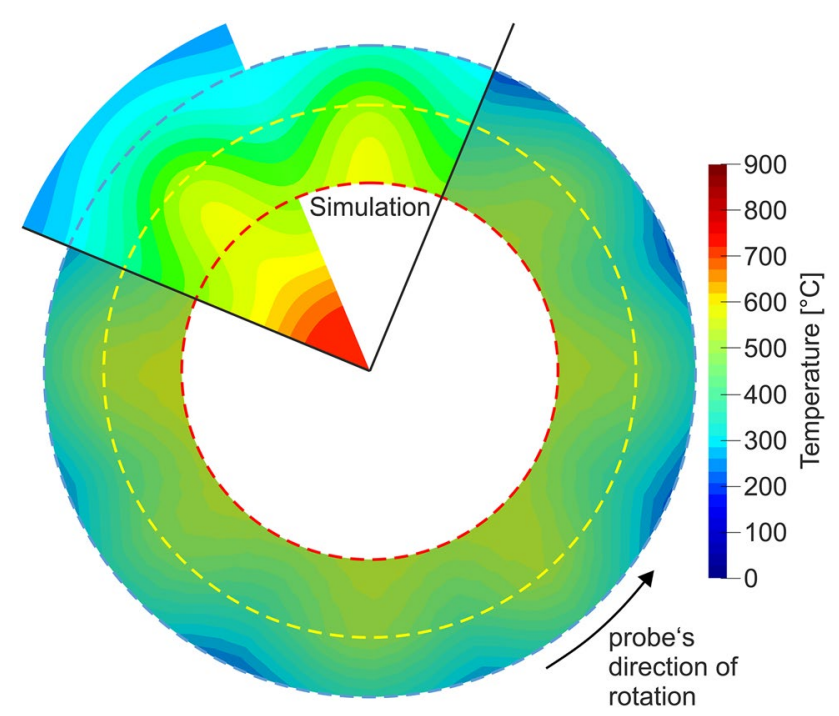

Fig. 17 Temperature distribution LMN (far field): measured vs simulation

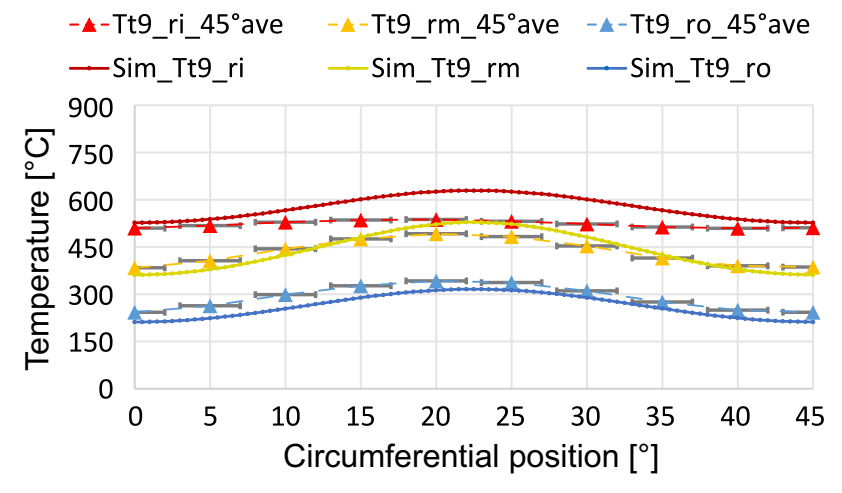

Fig. 18 Circumferential profile LMN (far field): measured temperature vs simulation static temperature

\subsubsection{Lobe mixer nozzle "scalloped" LMN-SC}

Figure 19 contains the measured temperature distribution at the outlet of the scalloped lobe mixer nozzle LMN-SC. For comparison, a $45^{\circ}$ segment of the static temperature distribution from the simulation is added.

The hot flow zone from the simulation is mirror-symmetrical, while the measured temperature distribution graph appears to have a dent on one side. This phenomenon is caused by the rotational direction of the measuring probe system. When the thermocouples move into the hot zone, the temperature is underestimated due to the measurement's inertia. A longer hold time before measurement would have been necessary here. Again, the limited resolution of the measurement makes it difficult to detect the exact position of the transition in the measured distribution. The plot of the circumferential profiles (Fig. 20) describes an interesting connection between simulation and measurement. The

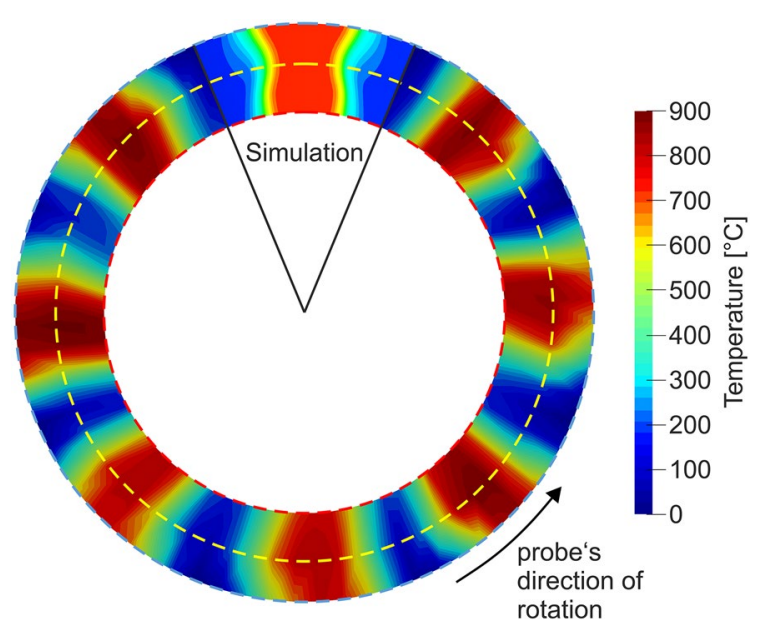

Fig. 19 Temperature distribution LMN-SC (near field): measured vs simulation 


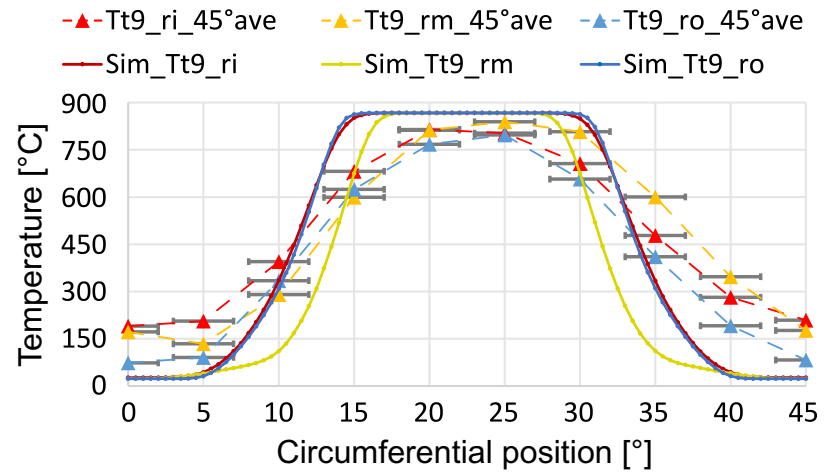

Fig. 20 Circumferential profile LMN-SC (near field): measured temperature vs simulation static temperature

temperature lines from the simulation of the LMN-SC are much closer together than on the LMN. The same mechanism can be found in the lines of measurement. The temperatures on the three measuring radii reach the same level in the middle of the hot zone in measurement and simulation.

The measured temperature towards the inner radius is higher in the cold zone. The ambient air heats up on the warm casing parts of the engine and the nozzle shroud. The inward lobes direct this air into the nozzle flow, a phenomenon that is not considered in the simulation.

The measurement of the far field of the LMN-SC (Fig. 21) achieved an excellent correspondence between simulation and experimental data. Both simulation and measurement confirm that mixing in the middle and outer areas is further improved by the LMN-SC. The simulation also indicates that the advanced geometry of the LMN-SC can shift more

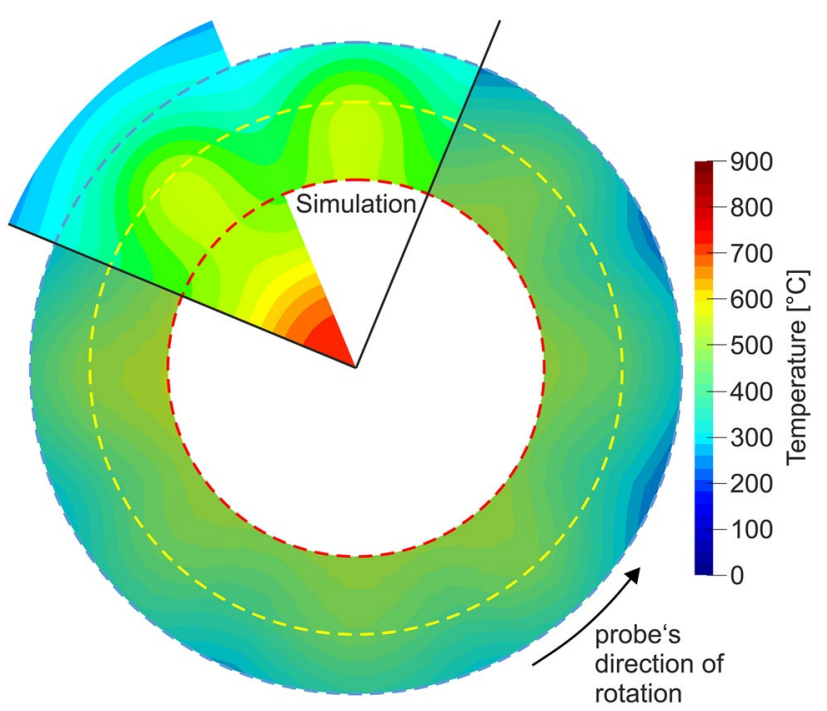

Fig. 21 Temperature distribution LMN (far field): measured vs simulation momentum from the core flow to the outer area than the normal lobe mixer nozzle does.

The circumferential profiles (Fig. 22) of the static temperature from simulation data tend to be close to the measured temperature profiles. The higher deviations at the middle radius $r_{\mathrm{m}}$ may be due to averaging the temperatures of all $45^{\circ}$ segments of the measurement. The scalloped lobe mixer nozzle LMN-SC achieves higher mixing speeds in simulation and measurement. The degree of mixing $\beta_{\text {mix }}$ is calculated as 1.248 using the simulation data.

\subsection{Shear layer angle of primary nozzles}

With the Schlieren optics, it is also possible to analyse the longitudinal conditions of the nozzle flow. The use of a highspeed camera provides detailed information on dynamic phenomena. The Schlieren optics utilises the relationship between density and the refractive index of a medium. Density gradients cause lighter or darker areas in the image. All the deflections of the light path are summed up. In the case of a rotationally symmetrical flow, this makes a direct comparison to the density distribution difficult. Only the rim area of the nozzle flow can be rated accurately.

Figure 23 shows the density distribution in a longitudinal section from the simulation and the Schlieren image of the standard nozzle SN. The simulation indicates a narrowing of the core flow to the end of the inner cone. Similar behaviour can also be seen in the Schlieren image. Behind the nozzle outlet, the shading is darker to the end of the inner cone. The shear layer angle largely coincides between the density distribution and the Schlieren image. This is determined to be $6.5^{\circ}$

Figure 24 shows the density distribution in the longitudinal section of the simulated chevron nozzle CVN and the experimental Schlieren image. According to the simulation diagram, the chevron nozzle's core flow also narrowed to the end of the inner cone. The video footage reveals a high

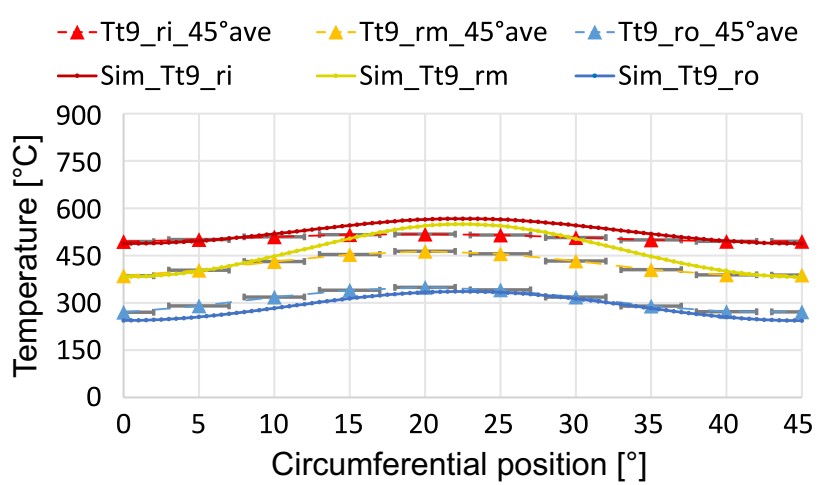

Fig. 22 Circumferential profile LMN-SC (far field): measured temperature vs simulation static temperature 


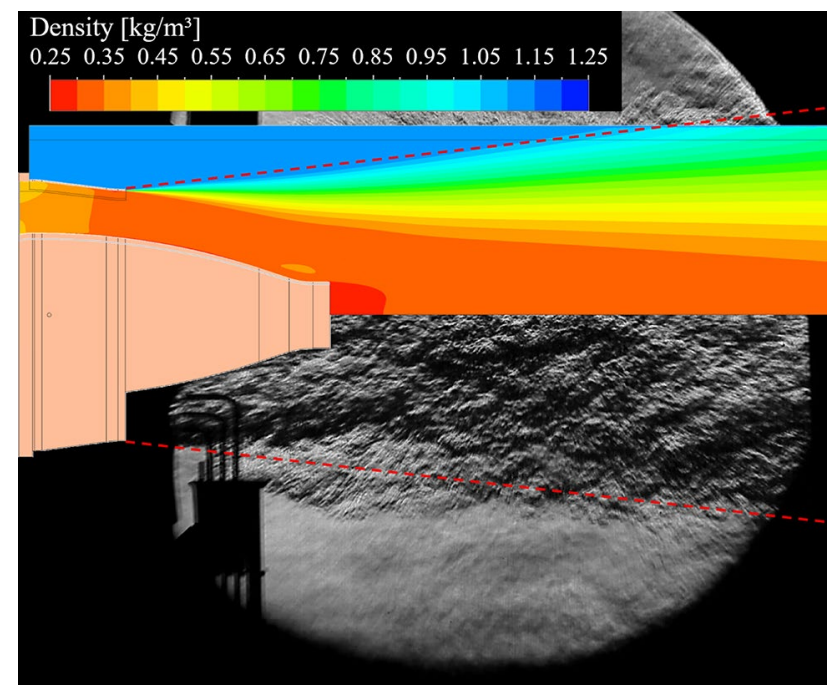

Fig. 23 Schlieren image vs density plot nozzle flow SN

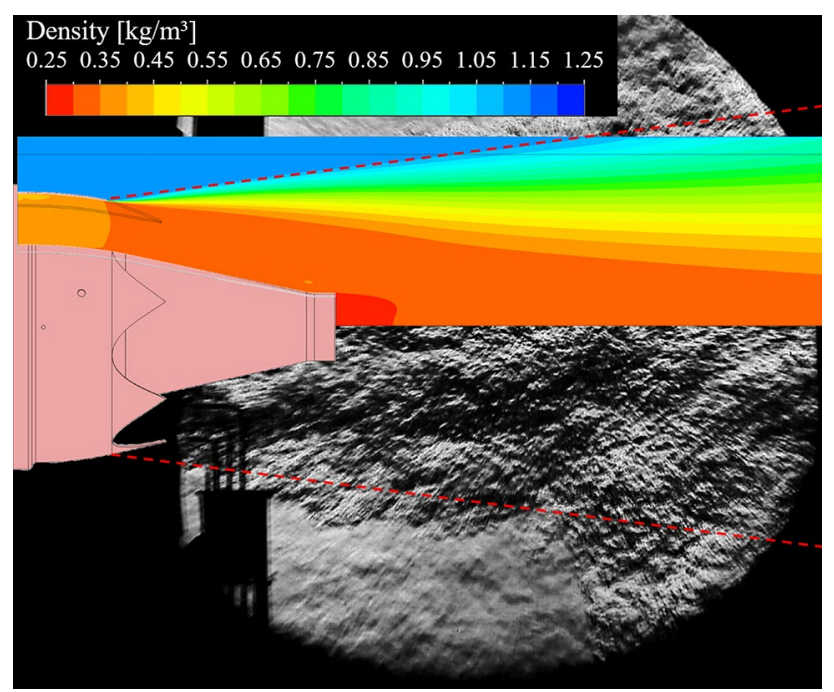

Fig. 24 Schlieren image vs density plot nozzle flow CVN

dynamic shear layer flow and vortex structures. The rotation of the vortexes appears to transport ambient medium into the nozzle flow. The shear angle is determined from the density distribution to a value of about $7^{\circ}-8^{\circ}$.

Figure 25 presents the density distribution of the lobe mixer nozzle LMN in a longitudinal section of the nozzle flow and the Schlieren image. The core flow is only slightly narrowed after exiting the lobe mixer nozzle. The hot core flow largely dispenses at a short distance behind the inner cone. In the Schlieren image, a pattern of darker and lighter stripes forms directly after exiting the nozzle. These match the tips of the lobes. The stripe pattern in

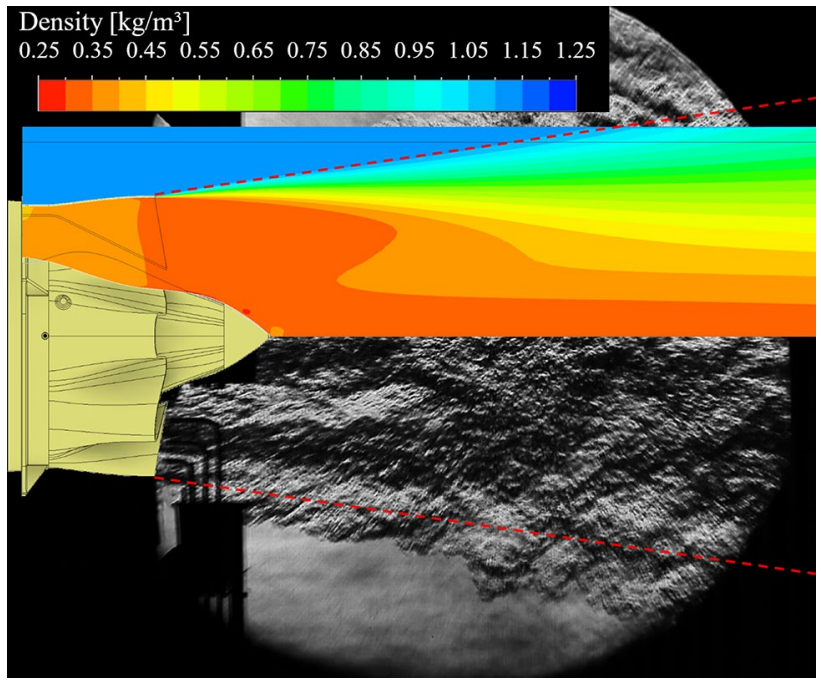

Fig. 25 Schlieren image vs density plot nozzle flow LMN

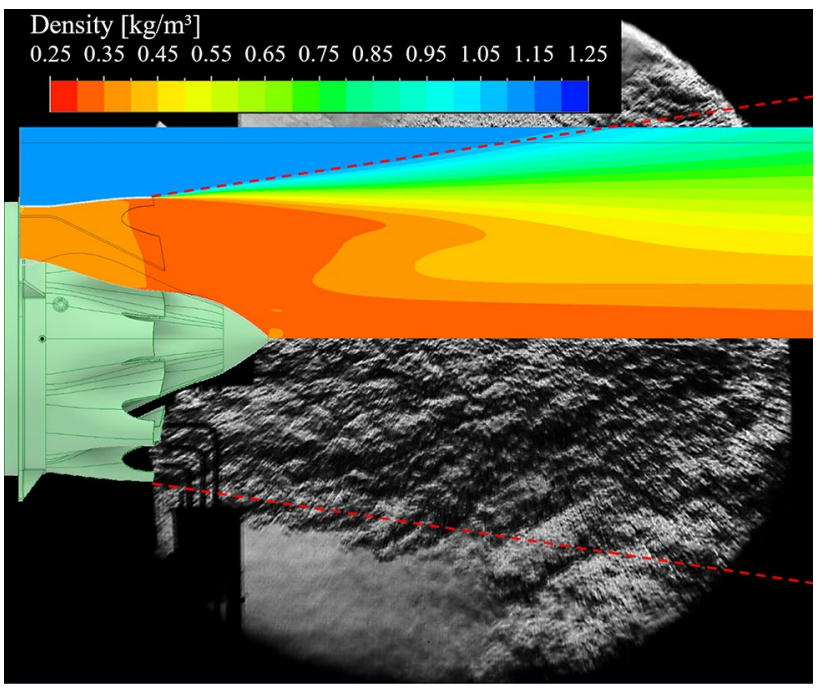

Fig. 26 Schlieren image vs density plot nozzle flow LMN-SC

the Schlieren image also stops at the same level on which the core flow dispenses in the simulation. According to the simulation data, the shear layer angle is approx. $8^{\circ}$.

The Fig. 26 shows the longitudinal distribution of the density from the numerical simulation results of the LMN$\mathrm{SC}$. This is compared with the Schlieren image from the same nozzle. The basic flow behaviour of the LMN-SC is the same as that of the LMN. Despite this the decomposition of the hot core flow starts earlier. This is confirmed by the much less pronounced stripe pattern in the Schlieren image. The shear layer angle is between $8^{\circ}$ and $9^{\circ}$. 


\subsection{Experimental results for the effect of the mixing ability on the thrust augmentation with ejector nozzle}

The identified mixing capabilities of the different nozzle types should influence the performance of the ejector nozzles. According to the theory, the achievable pumping rate is directly related to the degree of mixing $\beta_{\text {mix }}$. On the other hand, the thrust augmentation correlates with the secondary mass flow ratio (SMR) and the thrust efficiency of the primary nozzle.

$\mathrm{SMR}=\frac{\dot{m}_{E J}}{\dot{m}_{p}}=\frac{A_{\text {measure }} \cdot \rho_{\mathrm{EJ}} \cdot c_{\mathrm{EJ}}}{\dot{m}}$

The following performance values (Table 3) resulted from experimental measurement of the ejector nozzle configurations (Fig. 1). The uncertainty of the thrust measurement is approx. $1.5 \%$ and of the fuel mass flow about $0.5 \%$. The secondary mass flow ratio is affected by several uncertainties, such as blockage of the passage and velocity gradient. The error is approx. $20 \%$, so that statements can only be made about the relative change in the SMR. The nozzles' thrust efficiency is assessed qualitatively with three parameters compared to the standard nozzle SN: The relative difference between the density-corrected thrust and the thrustspecific fuel consumption TSFC as well as the determined shear layer angle (see Sect. 5.2).

The EJ8610-SN is set with a maximum secondary mass flow ratio SMR of 1.0 as a reference. The estimation of the SMR with the flow velocity in the secondary inlet actually comes to a mass flow ratio of 1:1. The thrust augmentation achieves $6.5 \%$. Due to the relatively low mixing, the diffuser leads to a loss of thrust. The thrust efficiency of the chevron nozzle is rated lower. The CVN has $2 \%$ less thrust and almost the same TFSC. The shear angle is slightly higher. Despite the higher $\beta_{\text {mix }}$ of the CVN, the EJ8610-CVN suffers a lower pumping rate with a relative SMR of 0.9. This leads to in a lower 5\% thrust augmentation, which has also been found in previous tests $[5,6]$. The induced mixing speed of

Table 3 Results of the ejector nozzle tests

\begin{tabular}{llll}
\hline Configuration & $\Phi_{\mathrm{F} \_r l_{1}}(\%)$ & TSFC $_{\text {rel }}(\%)$ & SMR \\
\hline EJ8610-SN & 6.5 & -5 & 1.0 \\
EJ8610DIF-SN & 5.0 & -4 & 1.2 \\
EJ8610-CVN & 5.0 & -4 & 0.9 \\
EJ8610DIF-CVN & 5.0 & -4 & 1.1 \\
EJ8610-LMN & 9.0 & -7 & 1.2 \\
EJ8610DIF-LMN & 9.0 & -7 & 1.4 \\
EJ8610-LMN-SC & 6.5 & -5 & 1.1 \\
EJ8610DIF-LMN-SC & 8.0 & -7 & 1.4 \\
\hline
\end{tabular}

the chevron nozzle is too low to benefit from the use of a diffuser.

The LMN lobe mixer nozzle achieved the same thrust values as the standard nozzle SN. With $2 \%$ better TSFC and only a slightly larger shear layer angle, the thrust efficiency of the LMN is rated a little higher than the SN's efficiency. The EJ8610-LMN gains high mixing rates and large secondary mass flow (relative SMR 1.2). This results in a relative thrust augmentation of $9 \%$. The diffuser can increase the pumping performance but cannot compensate for the additional losses, so the thrust augmentation remains $9 \%$. The LMN-SC cannot efficiently accelerate the flow due to the cut-outs. The thrust values are $2 \%$ lower than with the SN. The TSFC is the same as the LMN. The shear layer angle tends to be higher than the angle of the LMN. Despite the higher mixing capacity, the relative SMR of the EJ8610LMN-SC drops to 1.1. The lower thrust efficiency combined with less secondary mass flow results in a lower thrust augmentation of $6.5 \%$. But for the first time, the better mixing provides a positive effect of using a diffuser and increases the thrust augmentation to $8 \%$.

\section{Parameter variations for lobe mixer primary nozzles}

The experimental data from the previous tests points out, that the lobe mixer nozzle largely improves the ejector performance. The primary nozzles' thrust efficiency and mixing ability must be optimised first for a high-performance ejector nozzle. The large number of design parameters results in various possible combinations for a lobe mixer nozzle.

\subsection{Geometrical parameter variation primary nozzle}

Certain parameters are kept constant to ensure comparability, and only those are varied, that are considered to have a significant influence (Table 4). The total lobe angle $\varphi$ and penetration PEN results are already available in Ref. [16], which is why these parameters are not examined further here.

The paths $\beta_{\mathrm{III}}(\mathrm{x})$ and $r_{\alpha}(\mathrm{x})$ are adapted to the required surface course $A(x)$ of the nozzle. The nozzle variations are designed to keep the nozzle pressure ratio $\Pi_{D}$ as equal

Table 4 Varied construction parameter

\begin{tabular}{ll}
\hline Cutback angle & $\xi_{\text {cut }}$ \\
Number of lobes & $\Theta$ \\
Outlet shape (LL, PL, SL) & $\beta_{\text {III }}\left(x_{\text {end }}\right)$ \\
Contoured inner cone & $\mathrm{CC}$ \\
\hline
\end{tabular}


as possible. For this purpose, the exit area $A_{\mathrm{p}}$ and the transition to the lobe shape of each nozzle is iteratively adapted. Except for the version with a contoured inner cone (CC), all nozzle variations use the same rotationally symmetrical inner cone. A 3D model is created for each variation and transferred to the numerical simulation. Because of the symmetry, the simulation model only uses an angular segment in the size of $2 \Theta$ to reduce the computational effort. The physical model and the mesh setting are reused from the mesh independency study. The boundary conditions come from the experimental data of the tested lobe mixer nozzle LMN.

\subsection{Analysis of the parameter variation on mixing ability for the primary nozzle}

The intention of the evaluation is to analyse the influence of the construction parameters on the velocity profile distribution of the nozzle flow.

For optimal mixing the lobe mixer nozzle must provide a fast compensation of the flow profiles in the radial and in the circumferential direction. The velocity profiles are examined directly at the nozzle outlet (P9) and at a distance of one nozzle outer diameter (1DD) (Fig. 27). At plane $\mathrm{P}$, the radial profiles (mid, sym1, sym2) are formed from the wall of the inner cone to the nozzle outer radius $r_{\text {olend }}$. This is done in the middle of the angular segment and at the edges. The circumferential profiles are defined on the area-quartered radii $\left(r_{\mathrm{m}}, r_{\mathrm{o}}, r_{\mathrm{i}}\right)$ of the ring surface between the inner cone and the nozzle outer radius $r_{\mathrm{ol} \text {,end }}$. The radial profiles (mid, sym1, sym2) are drawn from the axis of rotation to the designed effective radius on the plane with the distance $2 \cdot r_{\mathrm{ol}, \text { end }}(1 \mathrm{DD})$. This corresponds to the inner radius $R_{\mathrm{M}}$ of the ejector tube. The circumferential profiles are again defined on the area-quartered radii $\left(r_{\mathrm{m}}\right.$, $\left.r_{\mathrm{o}}, r_{\mathrm{i}}\right)$ of the circular cross-section.

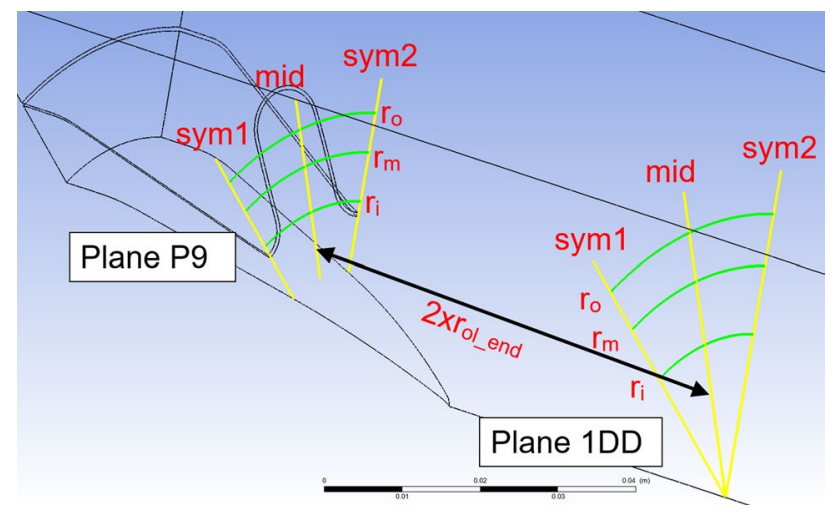

Fig. 27 Definition of evaluation planes, radii and lines

\subsubsection{Variation of the cutback angle}

A lobe mixer nozzle with eight lobes is constructed to evaluate the influence of the cutback angle $\xi_{\text {cut }}$ on the velocity profiles. Four different cutback angles $\xi_{\text {cut }}$ of $0^{\circ}$, $5^{\circ}, 10^{\circ}$, and $15^{\circ}$ are examined. The radial distribution of the velocity profiles of the four nozzle variants on plane 1DD is shown in Fig. 28.

The larger cutback angle $\xi_{\text {cut }}$ shifted the jet flow further towards the rim area. The core flow is not affected. The mixing $\beta_{\text {mix }}$ in plane 1DD increases slightly from 1.33 to 1.29 , but the overall impact is minor. The effect is mainly caused by the shortening of the lobe in the rim area, which increases the axial length of the mixing section.

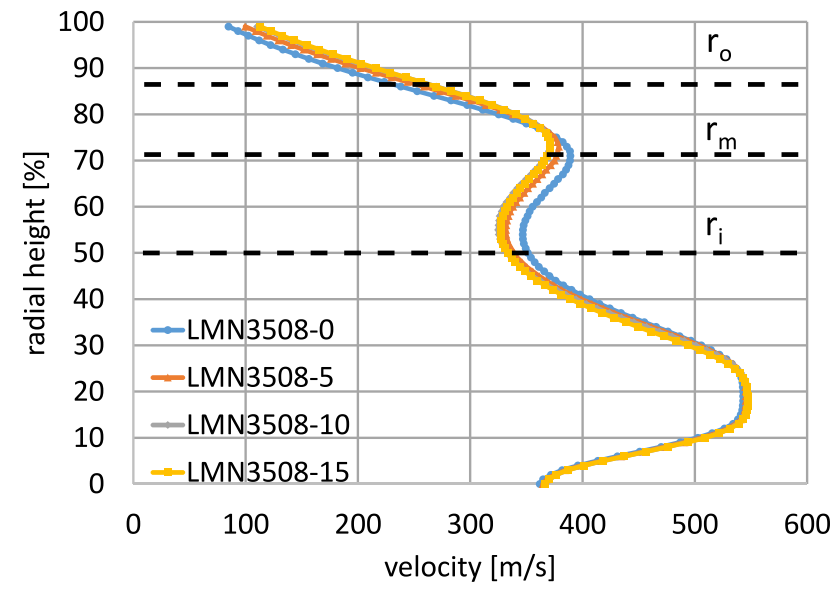

Fig. 28 Radial distribution of the flow velocity in plane 1DD on the middle line (mid)

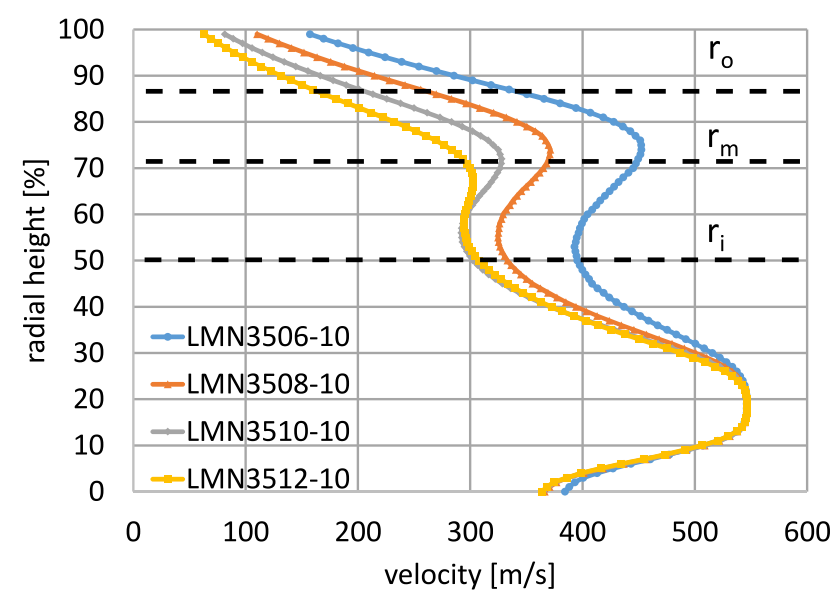

Fig. 29 Radial distribution of the flow velocity in plane 1DD on the middle line (mid) 


\subsubsection{Variation in the number of lobes}

Figure 29 contains the velocity profiles of four lobe mixer nozzles with $6,8,10$ and 12 lobes. The cutback angle $\xi_{\text {cut }}$ was kept constant at $10^{\circ}$. The outlet cross-section $A_{\mathrm{p}}$ was adjusted at each nozzle to sustain the same nozzle's pressure ratio $\Pi_{D}$. The increasing friction losses through the enlargement of the surface in the nozzle duct reduced the thrust. The variant LMN3512-10 with twelve lobes had $1 \%$ less thrust than the LMN3506-10 variant with six lobes. The radial distribution of the velocity on plane 1DD (Fig. 29) points out that the flow velocity decreases in the outer area with a higher number of lobes. The momentum is spread over more lobes. Therefore, the flow velocity gradient is equalised more quickly. The circumferential distribution of the flow velocity (Fig. 30) represents the difference in flow velocity between the outward and the inward lobe. This drops sharply when the number of lobes increases. The closer the lobes are to each other, the more the area between is affected, better equalisation can be achieved.

The lower velocity gradients can be quantified by improving the degree of mixing $\beta_{\text {mix }}$ (Fig. 31). The degree of mixing $\beta_{\text {mix }}$ improves up to the nozzle variant LMN3510 with ten lobes. After that, no further improvement in impulse compensation can be achieved. The radial distribution (Fig. 29) shows that the core flow is only marginally influenced by an increase in the number of lobes. The momentum of the core flow does not continue into the outer area. At a certain point, equalising the flow velocity in the outer area increases the gradient to the core flow. As a result, the degree of mixing of $\beta_{\text {mix }}$ cannot be further improved without more momentum being shifted to the outer area.

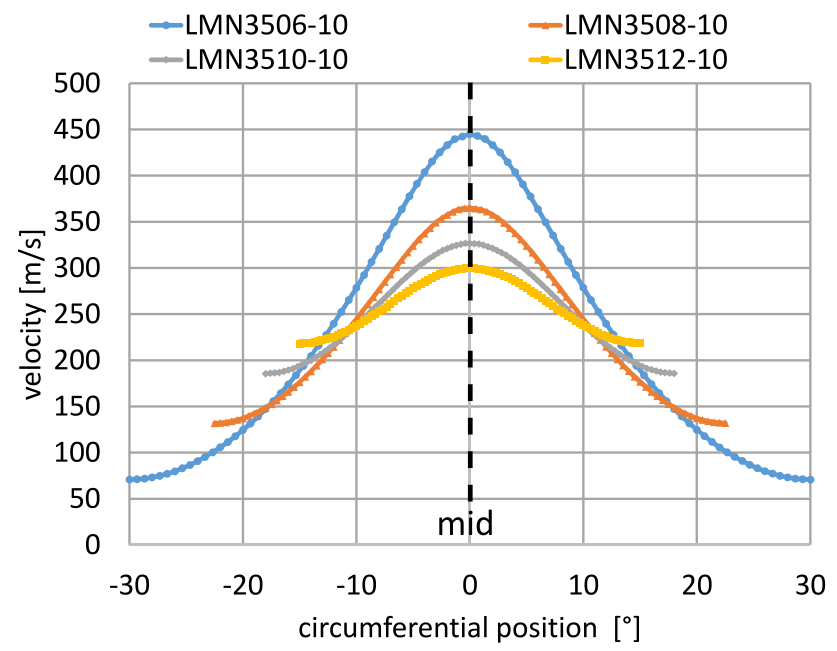

Fig. 30 Circumferential distribution of the flow velocity in the 1DD plane on the mean radius $r_{\mathrm{m}}$

\subsubsection{Loop lobe, parallel lobe and star lobe mixer}

Three lobe mixer nozzles with eight lobes each were constructed with different $\beta_{\mathrm{III}(\mathrm{x}, \mathrm{end})}$ angles to create the shapes of a loop, parallel and star lobe. The corresponding end angles $\beta_{\text {III(x,end) }}$ are $77.5^{\circ}, 67.5^{\circ}$ and $57.5^{\circ}$. The cutback angle $\xi_{\text {cut }}$ was kept at $10^{\circ}$ and the outlet area $A_{\mathrm{p}}$ was adjusted so that the same nozzle pressure ratio $\Pi_{D}$ is established. The result of the different outlet shapes is shown in the radial flow velocity profiles in Fig. 32. The lobe mixer primary nozzle LMN3508-10LL with a loop-shaped outlet contour achieves a significant shift in flow velocity from the middle to the outer area. The nozzle with a star-shaped outlet contour has a stronger velocity profile in the core flow area. The circumferential distribution directly on the nozzle outlet plane P9 shows that the loop-shaped lobe mixer already reaches a wide area of influence at the outlet. The star lobe mixer accordingly reaches a larger area of influence in the inner area.

The loop lobe mixer nozzle LMN3508-10LL performs best with a $\beta_{\text {mix }}$ of 1.26 . The parallel lobe mixer LMN3508-10 reaches 1.3 and the star lobe mixer nozzle

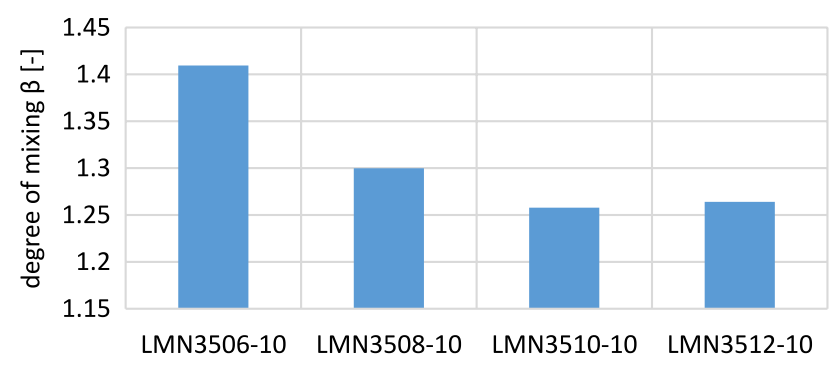

Fig. 31 Degree of mixing $\beta_{\text {mix }}$ depending on the number of lobes

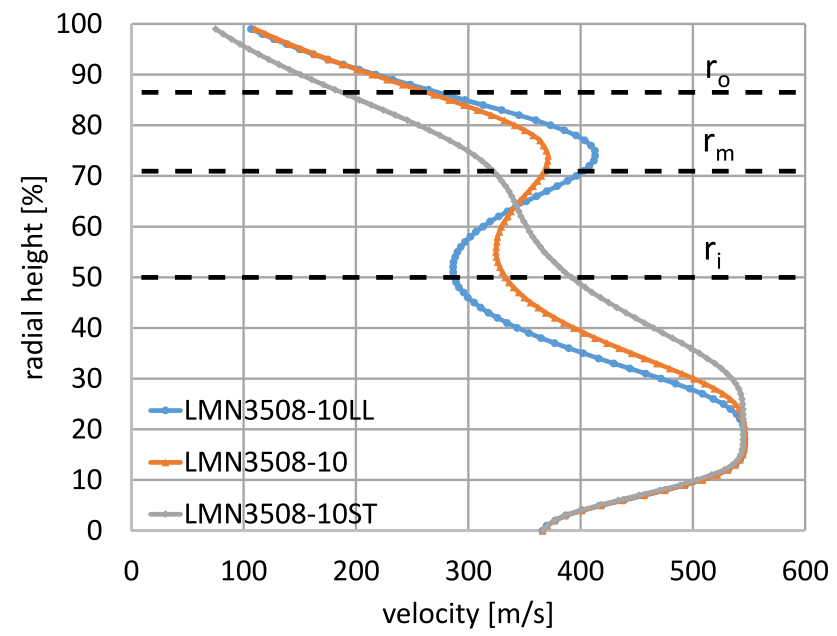

Fig. 32 Radial distribution of the flow velocity in plane 1DD on the middle line (mid) 
LMN3508-10SL achieves 1.4. For a beneficial speed equalisation, the flow impulse from the core flow must be shifted to the outer area. The more intense core flow from the starshaped contour degrades the degree of mixing.

\subsubsection{Influence of the contoured inner cone}

The lobe mixer nozzle LMN3508-10 was modified with a contoured inner cone (LMN3508CC-10) to assess the effect of a contoured inner cone on the velocity profiles. The radial velocity profile in plane 1DD is shown in Fig. 33. The inner cone's contouring made it possible to shift the core flow slightly to the outer area. The mechanism can also be found in the circumferential distribution in level 1DD. The maximal flow velocity on the inner radius $r_{\mathrm{i}}$ is reduced and increased in the middle and outer area. However, the degree of mixing $\beta_{\text {mix }}$ is approximately the same for both nozzles at 1.3. The contoured inner cone managed to shift the velocity impulse from the core flow to the outer area, but does not improve the mixing.

\section{Conclusion}

The study shows the great potential of ejector nozzles for small turbojet engines. The ejector nozzle concept enables thrust augmentation up to $9 \%$ and the exhaust gas temperature could be reduced significantly. The ejector nozzles combined with lobe-shaped primary nozzles could use to optimise engine performance. A new design process is developing these nozzles based on the requirements of the ejector nozzle and the engine.

Four types of primary nozzles were examined experimentally with a $180 \mathrm{~N}$ turbojet engine. The test campaign used

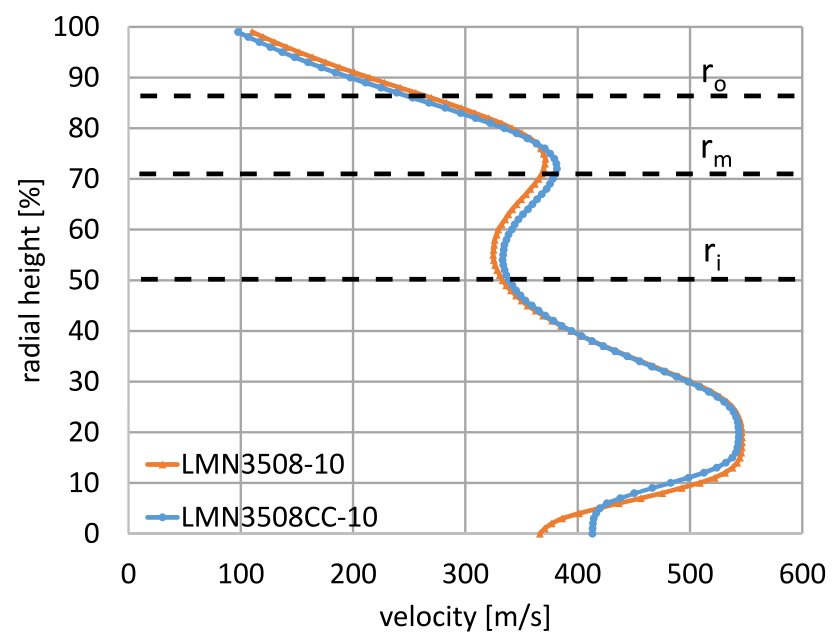

Fig. 33 Radial distribution of the flow velocity in plane 1DD on the middle line (mid) a standard ring cross-section nozzle $\mathrm{SN}$, a chevron nozzle $\mathrm{CVN}$, and two lobe mixer nozzles LMN and LMN-SC. The tests were continued with a combination of the different nozzle types with one ejector nozzle (see Fig. 1). A rotatable probe system was used to measure $360^{\circ}$ profiles of temperature $T_{\mathrm{t} 9}$ and total pressure $p_{\mathrm{t} 9}$. In parallel, a Schlieren optical system was operated to provide information about the longitudinal course of the nozzle flow.

The comparison of the results mostly reveals a good degree of agreement between numerical simulation and experimental measurement. The used method ensures a realistic reproduction of the nozzle flow of a small turbojet engine. Deviations are caused by the limited resolution of the measurement and the subsequent interpolation. Due to the strong temperature gradients in the nozzle flow, the positioning accuracy of the thermocouples plays a major role. The uncertainty in the temperature measurement, on the other hand, is hardly decisive.

The temperature distribution downstream the lobe mixer nozzles (LMN and LMN-SC) achieves excellent agreement between measurement and simulation in the far field, which is most relevant for evaluating the mixing ability.

The Schlieren optic reveals that the spikes of the chevron nozzle CVN create a vortex system and affect the mixing process. Since this is a transient phenomenon, it has not yet been integrated into the simulation. Consequentially, the simulation could underestimate the mixing ability of the CVN. On the other hand, the ejector nozzle could not benefit from the higher mixing ability of the CVN in the tests. It is assumed that the vortex systems cannot form in the ejector nozzle and that the mixing ability is therefore reduced.

The experimental investigations of the ejector nozzle also show an increased thrust augmentation due to the higher degree of mixing $\beta_{\text {mix }}$ of the lobe mixer nozzles. A $20 \%$ higher bypass is also achieved and, as a result, a better reduction in exhaust gas temperature. The diffuser generates another $20 \%$ more secondary mass flow, but only has a positive effect on the thrust, if the mixing is nearly complete. The authors conclude that the optimization of the primary nozzle is an essential prerequisite for the performance of the ejector nozzle.

Based on this, ten different nozzle variants were designed and examined in a numerical simulation. Various design parameters were modified. The effects of the different nozzles are evaluated using velocity profiles in different levels and the degree of mixing $\beta_{\text {mix }}$. The result of the variation and analysis demonstrates the influence of the nozzle shape on the mixing. The cutback angle $\xi_{\text {cut }}$ was increased from $0^{\circ}$ to $15^{\circ}$, whereby a slight improvement in the degree of mixing $\beta_{\text {mix }}$ from 1.33 to 1.29 is achieved through an increased momentum exchange in the rim area. However, the effect is rated a minor one. A significant improvement in $\beta_{\text {mix }}$ from 1.409 to 1.258 could be achieved by increasing the number of lobes from 6 to 10. More lobes improved the exchange of impulse, especially 
in the cold area between two lobes. The core flow remains mostly unaffected. A further increase in the number of lobes to 12 degrades $\beta_{\text {mix }}$ as the velocity difference to the core flow dominates the mixing quality. More lobes also lead to higher friction losses and poorer thrust. By changing the shape of the outlet cross-section, a further possibility was found to influence the balance of the flow profiles. A loop-shaped primary nozzle shows an increased flow intensity in the outer area, leading to an improved $\beta_{\text {mix }}$ of 1.26. In contrast, the star-shaped nozzle increases the flow impulse in the area close to the axis, but negatively impacted mixing, resulting in a $\beta_{\text {mix }}$ of 1.4. The analysis of the lobed primary nozzle's velocity profiles with a contoured inner cone shows a shift in the flow intensity from the area close to the axis to the outer area. However, this cannot improve the mixing and the $\beta_{\text {mix }}$ remains 1.3.

The results from the parameter variations conclude that modifications of the lobe form primarily change the velocity distribution in the middle and outer area. Until a certain limit it is particularly effective to use a combination of more lobes and a loop lobe exit contour. To further improve mixing, the impulse must be shifted outward from the core flow. The shape of the inner cone influences this mechanism. The numerical and experimental investigation findings will now enable the performance of the ejector nozzle to be optimised.

Funding Open Access funding enabled and organized by Projekt DEAL.

Open Access This article is licensed under a Creative Commons Attribution 4.0 International License, which permits use, sharing, adaptation, distribution and reproduction in any medium or format, as long as you give appropriate credit to the original author(s) and the source, provide a link to the Creative Commons licence, and indicate if changes were made. The images or other third party material in this article are included in the article's Creative Commons licence, unless indicated otherwise in a credit line to the material. If material is not included in the article's Creative Commons licence and your intended use is not permitted by statutory regulation or exceeds the permitted use, you will need to obtain permission directly from the copyright holder. To view a copy of this licence, visit http://creativecommons.org/licenses/by/4.0/.

\section{References}

1. Hirndorf, D., Hupfer, A., Erhard, W., Kau, H.-P.: Very small gas turbine jet engines - Current limits and potential for improvement. In: European Conference for AeroSpace Sciences (EUCASS), München (2013)

2. Hupfer, A.: Experimental investigation of performance of very small jet engine combustors. In: The 25th International Symposium on Transport Phenomena, Krabi (2014)

3. Hupfer, A., Hirndorf, D.: Investigation of parameters affecting thermodynamic cycle of very small jet engines. In: Proceedings of 15 th International Symposium on Transport Phenomena and Dynamics of Rotating Machinery (ISROMAC-15), Honolulu (2014)

4. Nelson, J., Dix, D.: Development of engines for unmanned air vehicles: Some factors to be considered. In: Institute for Defence Analyses, IDA Document D-2788, Alexandria (2003)
5. Schmidt, R., Hupfer, A.: Untersuchungen zu Ejektordüsen an kleinen Fluggasturbinen. Deutsche Gesellschaft für Luft- und Raumfahrt - Lilienthal-Obert e.V., urn:nbn :de:101:1-2018100511563581505119, Deutschland (2018)

6. Schmidt, R., Hupfer, A., Gümmer, V.: Thrust augmentation for very small jet engines using a mixer-ejector-system. In: 24th ISABE Conference 2019, Canberra, (2019)

7. Presz, Jr. W., Reynolds, G., Hunter, C.: Thrust augmentation with mixer/ejector systems. In: AIAA 2002-0230 40th AIAA Aerospace Sciences Meeting\&Exhibit, Reno (2002)

8. Presz, Jr. W., Gousy, R., Morn, B.: Forced mixer lobes in ejector designs. In: AIAA-86-161 AlAA/ASME/SAE/ASEE 22nd Joint Propulsion Conference, Huntsville (1986)

9. Presz, Jr. W., Werle, M.: Multi-stage mixer/ejector systems. In: 38th AIAA/ASME/SAE/ASEE Joint Propulsion Conference \& Exhibit, Indianapolis (2002)

10. Lord, W.K., Jones, C.W., Stern, A.M., Head, V.L., Krejsa, E.A.: Mixer ejector nozzles for jet noise suppression. In: 26th AIAA / SAE/ASME/ASEE Joint Propulsion Conference, Orlando (1990)

11. Porter, J.L., Squyers, R.A.: A summary/overview of ejector augmentor theory and performance volume 1. Vought Corporation, ATC Report No. R-91100-9CR-47A, Dallas (1979)

12. Presz, Jr. W., Reynolds, G., McCormick, D.: Thrust augmentation using mixer-ejector-diffusor systems. In: AIAA-94-0020 32nd Aerospace Sciences Meeting \& Exhibit, Reno (1994)

13. Rahn, D., Schmidt, R., Greif, F., Hupfer, A.: Untersuchungen zu Düsenströmungen kleiner Fluggasturbinen. Deutsche Gesellschaft für Luft- und Raumfahrt - Lilienthal-Oberth e.V.; Deutscher Luft- und Raumfahrtkongress URN: urn:nbn:de:101:1-201, Deutschland (2015)

14. Paterson, R.W.: Turbofan mixer nozzle flow field-A benchmark experimental study. J. Eng. Gas Turbines Power. 106, (1984)

15. Kozlowski, H., Kraft, G.: Experimental evaluation of exhaust mixer for an energy efficient engine. AIAA Paper 80-1088, Hartford (1980)

16. Presz, W., Blinn, R.F., Morin, B.: Short efficient ejector systems. In: AIAA/SAE/ASME/ASEE 23rd Joint Propulsion Conference, San Diego (1987)

17. Skebe, S.A., McCormick, D.C., Presz, W.M.: Parameter effects on mixer-ejector pumping performance. In: AIAA 26th Aerospace Sciences Meeting, Reno (1988)

18. Hilsenrath, J., Beckett, C., Benedict, W., Fano, L., Hoge, H., Masi, J., Nuttall, R., Toulaukian, Y., Woolley, H.: Tables of thermodynamic and transport properties of air, argon, carbon dioxide, carbon monoxide, hydrogen, nitrogen, oxygen and steam. Pergamon Press LTD., Oxford (1961)

19. ANSYS, Inc.: ANSYS CFX 14.5-Modelling guide (2012)

20. Blaisdell, A.S., Sullivan John, P., Gregory, A., Lyrintzis, L., Gregory, A., Lyrintzis: Preliminary design and computational analysis of an ejector nozzle with chevrons. School of Aeronautics and Astronautics S. 17, Purdue University (2011)

21. Kümmel W.: Technische Strömungsmechanik - Theorie und Praxis. Teubner Verlag, ISBN 978-3-8351-0141-8, Wiesbaden (2007)

22. Glawe, G.E., Simmons, F.S., Stickney, T.M.: Radiation and recovery corrections and time constants of several chrome-alumel thermocouple probes in high-temperature, high-velocity gas streams. NACA TN 3766, Washington (1956)

Publisher's Note Springer Nature remains neutral with regard to jurisdictional claims in published maps and institutional affiliations. 